\title{
CHIANG CHING-KUO (1910-1988): UNA VIDA INTERESANTE EN EL CORTO SIGLO XX CHINO ${ }^{2}$
}

\author{
Andrés Herrera Feligreras \\ Instituto de Historia Económica y Social Gerónimo de Uztáriz \\ a.herrera@herrerazhang.com \\ Yu-Ting Lu \\ Wenzao Ursuline University of Languages, Taiwan \\ 98013@mail.wzu.edu.tw \\ Ferran Pérez Mena \\ Universidad de Sussex, Reino Unido \\ F.Perez-Mena@sussex.ac.uk
}

RESUMEN: Chiang Ching-kuo (1910-1988) es una figura imprescindible en la comprensión del Mundo Chino contemporáneo. El presente artículo propone una aproximación a través de los capítulos más importantes de su vida, desde

1. El presente artículo forma parte de un proyecto de investigación que, bajo la denominación "Chiang Ching-kuo y el corto siglo chino (1910-1988)" fue presentado en la Universidad de La Rioja dentro del XIII Seminario del Grupo de Investigación de Historia de Nuestro Tiempo (GIHNT) 'Frontera'.

2. En este artículo, y como norma generalizada de transliteración, se utilizará el sistema pinyin de romanización de la lengua china. No obstante, existen casos en los que por su difusión histórica (Tratado de Nankín), su uso común entre hispanohablantes (Pekín o Nankín, en lugar de Beijing o Nanjing) o nombres propios como los de Chiang Kai-shek o Chiang Chingkuo (Jiang Jieshi y Jiang Jingguo en pinyin) usaremos las recomendaciones de la Real Academia Española o del sistema Wade-Giles. De igual manera, en la referencia a nombres chinos se seguirá la norma común, esto es primero el apellido. Así por ejemplo Chiang es el apellido y Kai-shek el nombre. Finalmente, y para favorecer la identificación de las personas e instituciones nombradas se hará referencia a su denominación en inglés o chino optando, en el caso de estas últimas, por el uso -tradicional o simplificado- del sujeto o la organización nombrada. 
su experiencia en la Unión Soviética hasta su presidencia de Taiwán durante la década de los años setenta y ochenta. La trayectoria vital de Chiang Ching-kuo permite asomarse al complejo nudo de relaciones existentes detrás del especifico proyecto modernizador chino que se cristalizó en la isla debido a una combinación de elementos exógenos y endógenos con un grado de contingencia no prevista por la élite política del Kuomintang. Este trabajo, que busca contribuir al desarrollo de los estudios chinos y taiwaneses en español, propone el concepto de "un corto siglo chino" y sugiere nuevas líneas de investigación conducentes a cuestionar el discurso dominante sobre el "milagro taiwanés".

Palabras clave: Chiang Ching-kuo, "corto siglo XX chino", Kuomintang, China, Taiwán.

\section{CHIANG CHING-KUO (1910-1988): AN INTERESTING LIFE IN THE CHINESE 20TH CENTURY}

ABSTRACT: Chiang Ching-kuo (1910-1988) is an essential figure to understand the contemporary Chinese world. This article examines the most important chapters of his life, from his experience in the Soviet Union until his presidency in Taiwan during the 1970s and 1980s. Chiang Ching-kuo's vital trajectory allows us to study the complexities behind the particular Chinese modernization project that materialised in the island as a result of the combination of both exogenous and endogenous elements and a degree of contingency that was not expected by the political elite of the Kuomintang. This work seeks to contribute to the development of the Chinese and Taiwanese studies in Spanish. It advances the concept of "Chinese short century" and opens up new avenues for future research that seek to cast into doubt the dominant discourse around the "Taiwanese miracle".

Keywords: Chiang Ching-kuo, "Chinese short century", Kuomintang, China, Taiwan.

Recibido: 21 de julio de 2020 Aceptado: 13 de noviembre de 2020

Resulta imposible comprender el Mundo Chino contemporáneo ${ }^{3}$ sin el estudio del Kuomintang (KMT). Su evolución ideológica y política, desde su gé-

3. Frente al término, directamente traducido del inglés, de Gran China, o región de Gran China para referirse a la República Popular China (China continental y sus regiones administrativas especiales de Hong Kong y Macao) y la República de China (Taiwán) como una única unidad, en este artículo se propone el concepto "Mundo Chino" en sustitución de la traducción directa del término "Greater China". Hablar de "Mundo Chino" como hablar de "Mundo 
nesis como organización revolucionaria hasta su constitución en uno de los principales partidos conservadores del mundo. De igual manera, se encuentran interesantes aportaciones sobre la figura de Chiang Ching-kuo (CCK), sin cuyo legado no es posible entender el Taiwán de nuestros días.

Lamentablemente no es el caso de España, donde los estudios chinos en general y taiwaneses en particular sufren de un importante retraso. En ese sentido, el primer objetivo de este trabajo consiste en contribuir a los estudios sobre Taiwán en español a través de una panorámica sobre la figura de CCK. Un esbozo biográfico que desmiente, a través de la incorporación de investigaciones que han trabajado sobre archivos soviéticos, la narrativa auspiciada desde cierto sector de la historiografía según la cual CCK fue un rehén de Stalin durante su estancia en la URSS. Asomarse a la política desplegada por CCK pone bajo sospecha el discurso sobre la naturaleza liberal del "milagro económico taiwanés" e, igualmente, desmitifica su papel como abanderado de la instauración de una democracia liberal en Taiwán. Pero, sobre todo, la panorámica histórica que ofrece la vida de Chiang Ching-kuo (1910-1988) posibilita poner sobre la mesa un concepto: el corto siglo XX chino.

Iván Berend acuñó el concepto de "corto siglo XX", después desarrollado por Eric Hobsbawm para referirse al periodo comprendido entre 1914 y 1991 , es decir, entre el comienzo de la I Guerra Mundial y el colapso de la Unión Soviética. Si bien el foco de Hobsbawm está centrado en Europa, los autores miramos hacia el Mundo Chino siguiendo esta idea y nos atrevemos a proponer la existencia de un "corto siglo chino" que discurriría entre la Revolución Xinhai (1911) y los cambios políticos y económicos sucedidos, a primeros de los noventa, en ambos lados del estrecho de Formosa ${ }^{4}$.

La aproximación a la trayectoria política y vital de CCK permite acercarse a la agitada historia de China durante el siglo XX. Caminar por el sendero de su vida, es hacerlo por un laberinto de decisiones que reflejan su conocimiento de la política como "el arte de lo posible" y explican porque CCK fue uno de los grandes supervivientes políticos del siglo XX. En definitiva, se tratan -como en el título de la autobiografía de Hobsbawm- de años interesantes.

\footnotetext{
Árabe" no solo hace referencia de una forma mucho más neutra a los territorios citados sino que además conlleva una connotación cultural que sobrepasa las fronteras políticas.

4. La idea del corto siglo XX (short twentieth century) es desarrollada por Hobsbawm en su trabajo The Age of Extremes: The Short Twentieth Century, 1914-1991, London 1995. publicado en castellano como Historia del siglo XX, 1914-1991. Barcelona 1995. Este concepto estaría contrapuesto al "largo siglo XIX" que a su juicio se iniciaría con la Revolución Francesa (1789) y tendría su fin en la Gran Guerra. En la página 10 de la sexta edición en castellano (2003) de esta obra, Hobsbawm deja constancia de su agradecimiento a Iván Berend, presidente de la Academia de Ciencias Húngara de Ciencias entre 1985 y 1990, por el concepto "siglo XX corto".
} 


\section{China en revolución (1911-1949)}

Cuando finalizaba el siglo XIX, la relación de China ${ }^{5}$ con las potencias occidentales, Rusia y Japón, estaba fundamentada tanto por las consecuencias derivadas de los Tratados Desiguales ${ }^{6}$ como en la actitud acomodaticia y pacificadora, hacia los extranjeros, puesta en práctica por la minoría gobernante manchú que no era siempre compartida por la élite china. Desde la Segunda Guerra del Opio (1856-1860), existía una alianza tácita de la aristocracia manchú con ingleses y franceses para apuntalar en el poder a la dinastía Qing ${ }^{7}$. Alianza que comenzó a fraguarse para asistir al poder imperial durante la Rebelión Taiping (1850-1864) y quedaba ejemplarizada en el Servicio Imperial de Aduanas Marítimas ${ }^{8}$. Un organismo dependiente oficialmente de los manchúes pero gobernado realmente por funcionarios extranjeros, principalmente británicos. El Servicio Imperial de Aduanas Marítimas supuso la modernización de los ingresos fiscales procedentes del comercio exterior pero también la intervención directa en las finanzas del Imperio Qing que, desde la firma del Protocolo Bóxer en 1901, se dedicaban en primer lugar a pagar las gravosas indemnizaciones de guerra a las potencias signatarias del tratado ${ }^{9}$. En la connivencia entre el círculo de poder imperial y las potencias extranjeras garantes del orden subyacía, tal y como ha señalado Fairbank, "una divergencia entre los intereses de la dinastía manchú y los intereses del pueblo chino, los que poco a poco empezaron a verse como dos asuntos separados"10. No es de extrañar, por tanto, el surgimiento de distintas organizaciones clandestinas de carácter revolucionario opuestas tanto a los Qing como al imperialismo. Algunos de estos grupos convergieron en la Alianza Revolucionaria China o Tongmenghui, una organización que combinaba objetivos republicanos, nacionalistas y socialistas. Esta alianza, encabezada por Sun Yat-sen, es quizás la organización más conocida, pero no era la única $y$, de hecho, la primera década del siglo XX constituye un archipiélago de levantamientos frustrados contra la dinastía gobernante.

En 1910, por tanto, China era un vasto territorio gobernado por una dinastía continuamente en jaque e intervenido por las potencias imperialistas. Ese mismo

5. Oficialmente Imperio del gran Qing (大清帝國).

6. Los Tratados Desiguales, el primero de ellos el Tratado de Nankín (1842), concedían diversos privilegios a los firmantes sobre el imperio Qing y se fundamentaban en dos principios básicos: libre comercio -con un arancel máximo del 5\% para las importaciones-y derechos de extraterritorialidad para aquellos extranjeros que violasen las leyes chinas.

7. Sobre el contexto que propició esta alianza informal entre la dinastía gobernante y las potencias extranjeras ver: Fairbank, J. K., China, una nueva historia. Barcelona 1996, pp. 284-287.

8. 大清皇家海關總稅務司

9. Para una panorámica sobre el rol de las Aduanas Marítimas ver: Brasó, C., "Las Aduanas Marítimas de China y el comercio sino-español, 1900-1930". Revista de Historia Industrial. Economía y Empresa 26, (2017), pp. 109-143.

10. Fairbank, J. K., China... p. 286. 
año nace, el 27 de abril en Fenghua (Provincia de Zhejiang), Chiang Ching-kuo (CCK) hijo de un matrimonio concertado entre Mao Fumei, una mujer tradicional y Chiang Kai-shek (CKS), en aquel momento un militar profesional que servía desde 1907 en Japón, que solo regresará a China en octubre de 1911 para unirse al movimiento revolucionario que acabaría con la dinastía Qing. Con su padre, CCK mantendría siempre una relación compleja y, en ocasiones, distante. Esa distancia filial se compensaría con los vínculos tejidos con su madre, una devota budista reconocida por su bondad. Cuando Mao Fumei murió asesinada en 1939, victima de un ataque aéreo intencionado contra su casa por la aviación japonesa, Chiang Ching-kuo hizo grabar sobre una piedra el juramento de vengar su muerte ${ }^{11}$.

La proclamación de la República de China (1 de enero de 1912) supone el inicio del siglo XX chino. Existen aspectos formales en este sentido -como la denominación de "China" por primera vez en la historia para nombrar al Estado, la adopción de la semana occidental de siete días o el establecimiento del calendario republicano, etcétera- pero son sobre todo las cuestiones de fondo, donde el movimiento revolucionario debe enfrentarse a las inercias de la historia y a sus propias tensiones internas, las que marcan el camino hacia las dos constantes que definen el siglo XX en el Mundo Chino: la modernización y la independencia ${ }^{12}$.

La derrota de la dinastía Qing y la instauración del poder republicano trajo consigo el colapso de la administración imperial abriendo una etapa de inestabilidad y revolución permanente que, con diferentes fases y características, no concluiría hasta 1949 con la proclamación de la República Popular China y el traslado del gobierno de la República de China a la isla de Taiwán. Todavía quedaban muchos años para eso, ahora, en 1922 el que fuera nombrado primer presidente de la República, Sun Yat-sen, Ileva una vida errante sometido a los continuos cambios de poder en la China dominada por los señores de la guerra. La Alianza Revolucionaria había dado paso al Partido Nacionalista Chino o Kuomintang (KMT), una organización cuyos miembros vinculados por lealtad a Sun se había mostrado incapaz de cumplir sus objetivos de unificación nacional. En 1923, todo cambió. En enero, tras dos años de contactos, se hacía público el compromiso soviético de ayuda al KMT; en febrero un nuevo golpe militar en Cantón posibilitó el regreso de Sun y la instauración de un gobierno que servirá de base para la reunificación de China.

11. El matrimonio entre CKS y Mao Fumei fue un desastre solo mantenido por la obediencia de CKS a su madre. Cuando esta falleció CKS inició los trámites de divorcio. No obstante, Mao Fumei siguió habitando en la casa familiar -lo que a la postre le costaría la vida- y CKS cubriendo su manutención. CCK mantuvo un constante contacto con su madre. En la piedra juramentada se lee "以血洗血” (lavar la sangre con sangre).

12. Sobre la cuestión nominal ver: Martínez-Robles D., "Reescrituras de la historia: China en las estrategias discursivas de la historiografía occidental". Gerónimo de Uztariz, 25 (2009), pp. 47-66. 
El Frente Unido, formado por el KMT y el Partido Comunista Chino (PCCh), con el asesoramiento de agentes de la Komintern, de la talla de Mijaíl Borodin y Vasili Blücher, financiación y el material soviético estuvieron en condiciones de mostrar al mundo sus avances en 1925. Ese año, tanto los éxitos en el frente militar -donde los ejércitos comandados por Chiang Kai-shek obtuvieron importantes victorias frente a los señores de la guerra del sur- como en el frente político -en el que nacionalistas y comunistas supieron canalizar el profundo impacto producido por el Incidente del 30 de mayo- favorecieron una corriente de optimismo patriótico que circulaba, principalmente, entre los jóvenes chi$\operatorname{nos}^{13}$. Para muchos de ellos, además, la Unión Soviética (URSS) -el único Estado que había reconocido al gobierno revolucionario de Cantón- era el espejo al que debía mirarse para liberar China de la opresión colonial y construir una sociedad más justa. Chiang Ching-kuo era uno de esos jóvenes y en el verano viajó hasta la Academia Militar de Whampoa para pedirle un permiso muy especial a su padre: quería ir a estudiar a la Unión Soviética ${ }^{14}$.

\subsection{Los "años rusos" (1925-1937)}

Cuando a finales de octubre, el joven Chiang abandonaba -rumbo a MoscúShanghái, su padre se había convertido en una figura central dentro del Kuomintang. Tras la muerte de Sun Yat-Sen (12 de marzo de 1925), los tres miembros más influyentes dentro del círculo de poder del KMT eran Liao Zhongkai -arquitecto de la alianza con los comunistas y referente de la facción pro-soviética dentro del Partido- Hu Hanmin, líder del ala derecha y Wang Jingwei, virtual sucesor de Sun y presidente, desde julio de 1925, tanto del Gobierno como del Consejo Político del Kuomintang. Técnicamente, Chiang Kai-shek, como comandante del Ejército Nacional Revolucionario (ENR), estaba supeditado al Consejo que presidía Wang. Sin embargo, el asesinato de Liao presumiblemente por orden de Hu, que acabó arrestado, fortaleció la posición política de CKS que, al fin y al cabo, tenía las armas bajo su mando. Pero el fraccionalismo dentro del Frente Unido iba mucho más allá. No solo se trataba de las tensiones entre ala izquierda y derecha del KMT, a su vez, fragmentadas en múltiples camarillas y grupos de interés; además, estaban las diferencias de agenda entre los nacionalistas y los comunistas, de los comunistas chinos entre sí y de la relación -siempre compleja- del PCCh con el Partido Comunista de la Unión Soviética (PCUS). Finalmente, conviene tenerlo presente, las discusiones en el seno de la Komin-

13. Para los años del Primer Frente Unido, su gestación y crisis una visión panorámica en Spence, J. D., En busca de la China Moderna. Barcelona 2011, pp. 385-468. Fairbank, China..., pp. 339-355.

14. Para los primeros años de Chiang Ching-kuo véase: Chiu, T., 蔣經國人格特質與臺灣政 治發展 (1972-1988) Chiang Ching-kuo's Personality Characteristics and Taiwan Political Development (1972-1988) [Tesis doctoral]. Taipei 2008, pp. 49-67. 
tern, el Partido y el gobierno soviético sobre la "cuestión china". En esta complejidad, las diferencias discurrían subterráneas bajo la bandera del sol blanco y las victorias del Ejército Revolucionario frente a los señores de la guerra. Ya en Moscú, desde finales de noviembre, Chiang Ching-kuo podía seguir con orgullo los éxitos de su padre continuamente alabados por los principales órganos de propaganda soviéticos ${ }^{15}$.

Chiang Ching-kuo destacó en la Universidad Sun Yat-sen de Moscú (UCTCh) ${ }^{16}$. No solo por ser el hijo del líder revolucionario chino. Su compromiso político lo llevaron a formar parte de la Liga de la Juventud Comunista de China y del Komsomol ${ }^{17}$. Karl Radek, rector de la universidad, pronto vio el potencial del CCK. Radek, militante bolchevique de primera hora formaba entonces parte de la denominada Oposición de Izquierdas que lideraba Trotsky. El ascendente de Radek, y otros profesores de la UCTCh, sobre los estudiantes favorecieron la identificación de estos con las posiciones trotskistas. CCK se integró -desde la convicción ideológica- en este colectivo. Sin embargo, cuando romper con el trotskismo se convirtió en una condición para ingresar en el Academia PolíticoMilitar Tolmachev ${ }^{18}$, fue el primero de los estudiantes chinos en abandonarlo ${ }^{19}$ demostrando un pragmatismo, una comprensión de la correlación de fuerzas y una lectura del momento político concreto que le acompañarían durante toda su vida. En enero de 1926, otro joven estudiante chino, que terminaría siendo compañero y amigo de Chiang Ching-kuo, llegó a la universidad. Su nombre: Deng Xiaoping.

El año 1926 parecía traer consigo buenos augurios para el ala izquierda del movimiento revolucionario chino. El II Congreso del Kuomintang (Guangzhou

15. En el análisis del periodo soviético hemos tomado como guía los capítulos de Taylor dedicados a la vida de Chiang Ching-kuo en la URSS, sin embargo han sido los trabajos de Pantsov y Shimoda, fundamentados en los archivos del PCUS y la Komintern los que han permitido más matices en nuestro trabajo. Ver: Taylor, J., The Generalissimo's Son: Chiang ChingKuo and the Revolutions in China and Taiwan. Cambridge 2009, pp. 49-73; Pantsov, A., The Bolsheviks and the Chinese Revolution 1919-1927. London 2013; Shimoda, K., "Chiang ChingKuo's 'Desired' Return to China in 1937. Viewed from the Document of the USSR". Journal of the Graduate School of Asia-Pacific Studies 31 (2016), pp. 19-38.

16. La traducción de su nombre oficial sería Universidad Comunista Sun Yat-sen de los Trabajadores de China, siendo conocida por KУTK por sus siglas en ruso, que hemos transliterado como UCTCh.

17. Acrónimo en ruso de Unión Comunista de la Juventud, era la organización juvenil del PCUS.

18. Posteriormente renombrada, Academia Político-Militar Lenin, su función era la formación política de los oficiales del denominado (hasta 1946) Ejército Rojo de Obreros y Campesinos.

19. Según uno de sus compañeros en el grupo de la Universidad "la idea de una actividad trotskista activa simplemente le aterrorizaba" en Pantsov, A., "From Students to Dissidents. The Chinese Trotskyists in Soviet Rusia", https:/www.marxists.org/history/etol/writers/pantsov/1994/china/studiss3.html (último acceso: 15/07/2020). 
4 a 19 de enero) había situado a los comunistas y militantes nacionalistas afines en los puestos claves del KMT ${ }^{20}$. Sin embargo, una serie de acontecimientos -de motivaciones todavía hoy confusas- se fueron encadenando a lo largo del año y escorando la posición de Chiang Kai-shek que terminó liderando el ala derecha del Kuomintang y propiciando, en complicidad con el crimen organizado y las grandes fortunas, primero la Masacre de Shanghái (12 de abril de 1927) y, seguidamente, la persecución, arresto y asesinato de comunistas a gran escala en las áreas controladas por el Kuomintang.

Los sucesos de Shanghái fueron una conmoción para los estudiantes chinos en Moscú y gasolina para la discusión en el seno del PCUS y la Komintern sobre cual era la línea correcta en la "cuestión china". El núcleo dominante, liderado por Stalin, continuó apostando por la alianza con el KMT bajo el argumento de que el gobierno nacionalista de Wang Jingwei -instalado en Wuhan desde principios de 1927- y los puestos clave en el Partido seguían bajo personas afines a la colaboración con los comunistas y la Unión Soviética. Los hechos demostrarían la falta de conocimiento que Moscú -tanto la línea oficial como la oposición trotskista- tenía sobre la realidad china. En mayo, Wang buscaría la reconciliación con Chiang Kai-shek y el Frente Unido quedaba definitivamente roto. El 1 de agosto, como respuesta a la represión iniciada en abril por CKS y al acercamiento entre Wuhan y Shanghái, el ala izquierda del KMT coaligada con el PCCh tomaban Nanchang. Había comenzado la Guerra Civil China.

En la narrativa de los "años rusos" de Chiang Ching-kuo (1925-137) existe, a partir de este punto, cierta diferencia entre el relato más difundido y lo ocurrido en realidad. El argumento más popular recoge que CCK solicitó volver a China pero Stalin no se lo permitió, reteniéndolo como un rehén para negociar futuros acuerdos con Chiang Kai-shek y exilándolo a Siberia por sus simpatías con el trotskismo. Esta idea surge de una literatura -en muchas ocasiones próxima al Kuomintang-que toma como principal referencia las memorias que CCK escribió, por indicación de su padre, tras su regreso a China en $1937^{21}$.

Diez años antes, tras la ruptura del Frente Unido, 50 jóvenes chinos miembros del KMT, entre los que se encontraba CCK, decidieron quedarse en la URSS. Así nos lo indican los investigadores que han trabajado con los archivos soviéticos y de la Komintern. Según estas fuentes, durante sus "años rusos" Chiang Ching-kuo fue un buen estudiante -tanto en la universidad, la academia político-militar y en el postgrado de ingeniería- se mantuvo en la "línea correcta" (primero se desmarcó políticamente de su padre, después del trotskismo)

20. Por indicación de la Komintern los miembros del PCCh se integraron en el KMT y mantenían doble afiliación por lo que eran elegibles para cargos de responsabilidad dentro de la organización nacionalista.

21. Un buen ejemplo de este punto de vista en Marks, T. A., Counterrevolution in China: Wang Sheng and the Kuomintang. New York 2014, p. 36. 
y mostró un buen desempeño profesional. De hecho, al mismo tiempo que algunos de sus antiguos camaradas de la oposición trotskista -o ex miembros como él- fueron represaliados durante la Gran Purga (1936-1938), CCK trabajaba en línea con los objetivos nacionales de la URSS, se implicaba en los órganos de propaganda soviéticos, llegaba a ser miembro del Partido Comunista de la Unión Soviética e incluso disfrutó de vacaciones en Crimea y otros privilegios reservados a los cuadros y dirigentes del Partido y el Estado ${ }^{22}$. La única mención al "exilio siberiano", que comúnmente es interpretada como el paso de CCK por un campo de trabajo forzado, se encuentra en sus memorias. Es más, Taylor sostiene que, tras caer enfermo a su llegada a Sverdlovsk ${ }^{23}$, las autoridades probablemente enviasen a CCK a Altái, en Siberia, no como castigo, sino para favorecer su recuperación sacándolo de la contaminada atmósfera de Sverdlovsk. De hecho, tras regresar a los Urales se le asignó responsabilidades de confianza en el complejo URALMASH, por aquel entonces el más grande de Europa, orgullo y emblema de la industrialización soviética. Según el propio Chiang, los funcionarios soviéticos trataron siempre de protegerlo del acoso de Wang Ming, líder de la fracción más ortodoxa del PCCh denominada "Veintiocho Bolcheviques" ${ }^{24}$.

Tras el Incidente de Mukden (18 de septiembre de 1931) y la consiguiente ocupación japonesa de Manchuria, Stalin consideró una nueva política hacia China que permitiese fraguar una alianza de contención frente a Japón. En di-

22. La trayectoria vital y profesional de CCK en la URSS no es la del represaliado por el terror estalinista que cierta historiografía sostiene, de hecho, en 1937 cuando la Gran Purga estaba en apogeo, la membresía de CCK en el PCUS fue aprobada, aunque al coincidir con las instrucciones de regresar a China no fue ratificada por el comité superior. Según Pantsov la trayectoria de CCK en la URSS se resume de la siguiente manera: Chiang Ching-kuo (alias Nikolai Vladimirovich Elizarov). Miembro de la Liga de la Juventud Comunista China desde 1925. Miembro del Komsomol soviético desde 1926. Miembro candidato de PCUS (19301941). Estudió en la UCTCh (1925-1927) y en la Escuela Militar Especial y en la Academia Político-Militar de Tolmachev (1927-1930). Trabajador metalúrgico en la planta de Moscú Dinamo (1930-1931). Presidente de la Granja Colectiva de la Revolución de Octubre en Korovino (provincia de Moscú), 1931. Estudiante de posgrado de la Escuela Internacional Lenin (19311932). Ayudante del jefe de taller del complejo de URALMASH, Sverdlovsk (1932-1934). Subdirector del periódico del complejo Uralmash Za Tyazheloye Mashinostroyeniye (1924-1927). Subdirector del Departamento de Organización Soviética de la ciudad de Sverdlovsk, 1937. Pantsov, The Bolsheviks..., posición. 7530 (edición Kindle).

23. Actual Ekaterimburgo. Allí también contrajo matrimonio, en 1935, con una destacada miembro local de las Juventudes Comunistas de la Unión Soviética, Faina Epatcheva Vahaleva. Faina -que en China y Taiwán se le conocería como Faina Chiang Fang-liang (蔣方良) mantuvo un discreto perfil público alejado de los focos de la política. Un perfil sobre su vida en Wu, P., "Forgotten first lady served as model traditional wife". Taiwan Times, 18 de mayo de 2003. http://www.taipeitimes.com/News/taiwan/archives/2003/05/18/0000211042 (último acceso 29/10/2020).

24. Sobre los "años siberianos" de CCK y la protección de los funcionarios del PCUS frente al acoso de Wang Ming ver: Taylor, The Generalissimo's Son..., pp. 61-62. 
ciembre de ese mismo año, Stalin busca ya la forma de enviar a CCK junto a su padre, algo que solo ocurriría después de que, en diciembre de 1936, el Incidente de $\mathrm{Xi}^{\prime}$ an propiciase una nueva alianza entre nacionalistas y comunistas frente a la ocupación japonesa. Entre 1931, cuando Stalin Ilama por primera vez a Chiang Ching-kuo - para conocer su perspectiva sobre la situación en China y compromiso para favorecer un nuevo Frente Unido entre el KMT y el PCCh frente a Japón-, y la fecha de su regreso a China (marzo de 1937), CCK fue consultado, en distintas ocasiones, tanto por Stalin como por la Komintern sobre la política hacia China.

El 28 de marzo de 1937, Chiang Ching-kuo, desde Sverdlovsk y antes de emprender su viaje de regreso a China, envió el siguiente telegrama a Dimitrov -Secretario General de la Komintern- "Te envío mis más sinceros saludos bolcheviques desde el camino. Todas tus instrucciones se llevarán a cabo" ${ }^{\prime 25}$. Tanto su padre, Chiang Kai-shek, como la dirigencia soviética -que consideraba a CCK un activo en la promoción de un Segundo Frente Unido antijaponés- querían el regreso de CCK a China. Como concluye Kimiko Shimoda "su regreso fue 'deseado' por ambos lados, pero al mismo tiempo ambos tenían expectativas diferentes" ${ }^{\prime 26}$.

\subsection{De regreso a China (1937-1949)}

El retorno de CCK a China estuvo marcado, tras el encuentro con su padre y por indicación de este, de un primer periodo de resinización: lectura de los clásicos, perfeccionamiento de su chino -especialmente la escritura- y puesta al corriente de la realidad en la que estaba inmersa China.

En 1937, China era un inmenso y fragmentado territorio en el que las pretensiones del gobierno republicano colisionaban con los señores territoriales, los intereses de las potencias occidentales, los de la URSS -especialmente en Mongolia y Xinjiang- y la grave amenaza del militarismo japonés que en 1932, tras la ocupación de Manchuria, había creado el estado títere de Manchukuo. La prematura muerte de Sun Yat-sen no permitió un desarrollo elaborado de su teoría revolucionaria y su esquema básico de tres fases (unificación militar, tutela política y gobierno constitucional) junto a la idea de partido-estado (Dang-Guo) convertían al KMT en un gran "barco del tesoro" capaz de albergar interpretaciones, incluso contradictorias, sobre lo que necesitaba China. Durante los diez años anteriores (1927-1937), el principal impulso modernizador vino de las élites urbanas y de la cooperación entre Nankín y Berlín en el ámbito económico y militar. Rechazado el modelo soviético que había inspirado a Sun, la búsqueda

25. Banac, I., The Diary of Georgi Dimitrov (1933-1949). New Haven 2003, p. 36.

26. Esta es precisamente la tesis de la que parte el trabajo de Shimoda en Shimoda, "Chiang Ching-Kuo's... 
de alternativas ideológicas en los que apuntalar el proceso de construcción de un Estado fuerte llevó a sectores del KMT a encontrar en el fascismo una fórmula, a su juicio más cercana, con los valores chinos tradicionales. La Sociedad de Camisas Azules o Linxingshe (1932) fue el instrumento organizado de aqueIlos que consideraron la vía fascista en China ${ }^{27}$. Sin embargo, al competir con la CC Clique ${ }^{28}$ por la atención de Chiang Kai-shek, su fundación no hizo sino fraccionar el círculo cercano a Chiang Kai-shek. Finalmente, el Movimiento de la Nueva Vida (1934) pareció ser la respuesta a la búsqueda de ese catalizador ideológico que permitiera a China, en palabras de Soong Mei-ling, "recuperar su posición como una gran nación" bajo el liderazgo de CKS en el partido y el Estado $^{29}$.

En este marco, existía un punto de consenso entre las camarillas que dominaban el Comité Ejecutivo del KMT: la modernización debía impulsarse desde arriba fundamentada, al menos superficialmente, en los valores tradicionales chinos $^{30}$. En esta "contrarrevolución moderna", como la definió Dirlik ${ }^{31}$, fue en la que debió integrarse CCK a su llegada a China e ingresar en el KMT. Una atmosfera que debió contrastar con un acervo ideológico, cultural y vital donde la pulsión transformadora se situaba en la base social compuesta por obreros y campesinos. Un paradigma, antagónico al imperante en Nankín, que tuvo su manifestación práctica a partir de 1938, cuando pasó a servir -primero en tareas relacionadas con seguridad, reclutamiento y administración- en la Provincia de Jiangxi.

Podemos especular aquí con la influencia de Radek en la formación de la visión política de Chiang Ching-kuo. Para el bolchevique, que como ya se ha

27. Para una panorámica sobre el fascismo en China ver: Chen, C., "La revolución fascista china: una alternativa de defensa del país antes del estallido de la Segunda Guerra Sino-Japonesa", Herrera-Feligreras, A., et alii, España y China 1937-2017. 80 aniversario del Internacionalismo Antifascista. Granada 2017, pp. 35-54 y Chen, C., Radicalización del nacionalismo chino moderno: orígenes y desarrollo del fascismo chino. El caso de las organizaciones fascistas del Guomintang: la Sociedad Lixingshe y el Movimiento de la Nueva Vida (1927-1937), Barcelona 2014.

28. Central Club Clique (中央俱樂部組織). Esta facción, liderada por los hermanos Chen Goufu y Chen Lifu, y considerada la extrema derecha más tradicional del KMT, configuraba la medula espinal del poder en el partido-estado especialmente durante la década de Nankín (1927-1937).

29. Li, L., Madame Chiang Kai-shek: China's Eternal First Lady. New York 2006, pp. 101-102.

30. En torno a la relación entre fascismo, modernidad, valores tradicionales y movilización de masas durante la década de Nankín ver: Griffin, R., Modernismo y fascismo. La sensación de comienzo bajo Mussolini y Hitler. Madrid 2010; Wakeman F., "A Revisionist View of the Nanjing Decade: Confucian Fascism". The China Quarterly 150 (1997), pp. 395-432 y Culp, R., "Rethinking Governmentality: Training, Cultivation, and Cultural Citizenship in Nationalist China". The Journal of Asian Studies 65 (2006), pp. 529-554.

31. Dirlik, A., "The Ideological Foundations of the New Life Movement: A Study in Counterrevolution". The Journal of Asian Studies 34 (1975), p. 946. 
comentado mantuvo una relación cercana con CCK, la toma de partido de los soldados (no la alta oficialidad) fue un factor disruptivo en el proceso revolucionario. El despertar de la conciencia de clase entre los uniformados -de extracción popular movilizados durante la I Guerra Mundial-fue determinante, y mayor que el rol jugado por obreros, campesinos y pequeña burguesía, en la formación de los soviets y posteriormente en la victoria de la Revolución ${ }^{32}$. Esta idea, sobre la que queda mucho por trabajar, podría explicar el especial cuidado que tuvo CCK por mejorar las condiciones de los soldados, o funcionarios de extracción popular, bajo su mando ya sea como reclutador para del Ejercito Nacional Revolucionario, en la Liga Juvenil ${ }^{33}$ o como responsable político del Ejercito Juvenil ${ }^{34}$.

Especulaciones aparte, algunos de los rasgos forjados durante su experiencia soviética como una visión igualitaria de la sociedad, el trato cercano, su desempeño como funcionario capaz y el rol del pueblo en la construcción de un futuro para China formarán una constante que le acompañarán de por vida. Una constante que, sin embargo, estará supeditada a la correlación de fuerzas existente en cada momento. Su discurso socialista se fue modulando con el paso de los años en respuesta a la evolución de la alianza del KMT con el PCCh, la relación de China con la URSS, y las tensiones intra-partidarias.

Desde su llegada, y siempre que tuvo ocasión de hacerlo, CCK mantuvo un discurso de abierto respaldo a la colaboración con el PCCh y la Unión Soviética que, al fin y al cabo, era la única potencia que ayudaba a China en su guerra contra Japón. Sin embargo, pronto comprendió que existía una pugna por la hegemonía entre los dos grandes partidos chinos. La creciente tensión entre ambas formaciones alcanzó su cenit con el Incidente del Nuevo Cuarto Ejército (01/1941) que puso fin, de facto, al Segundo Frente Unido. Meses después, la firma del Pacto de Neutralidad Soviético-Japonés (13/04/1941), terminó de dibujar para CCK un nuevo escenario que lo acercó, aún más, a su padre: el futuro de la revolución dependía del rumbo y ejemplo que fuera capaz de trazar y transmitir el KMT, la Unión Soviética solo era una potencia preocupada por sus propios intereses y los comunistas chinos un enemigo interno que había, como mínimo, que contener ${ }^{35}$.

Al igual que lo había hecho en la URSS, CCK se reveló como un burócrata eficaz que competía, con éxito, con los comunistas chinos por el apoyo tanto de las clases medias como de los sectores más populares. Para ello, frente a la corrupción imperante en una administración republicana cómplice de te-

32. Esta idea se desarrolla en: Radek, K., Las vías y las fuerzas motrices de la Revolución Rusa. Madrid 1976.

33. 三民主義青年團/三青團

34. 中國青年軍/中國青年軍遠征軍

35. En esta misma línea Taylor, The Generalissimo's Son..., p. 103. 
rratenientes, triadas y élite empresarial, CCK impulsó, durante su periodo en Gannan (1939-1944) ${ }^{36}$, una administración capaz de hacer frente a los males de la población, proporcionando trabajo e instrucción al tiempo que combatía la criminalidad y el mundo del hampa. Sus métodos de escuela soviética no gustaban en la élite del KMT que trataron de encorsetarlo sometiéndolo a una continua vigilancia. La respuesta de Chiang Ching-kuo fue construir una base de poder en el seno de la recién creada Liga Juvenil de los Tres Principios del Pueblo (YLTPP, por sus siglas en inglés).

A partir de la creación de la Liga en el Congreso Extraordinario del KMT (1938), CCK con su discurso antifascista y la promoción de la Liga como una organización con amplia autonomía respecto al KMT se ganó la animadversión tanto de los antiguos Camisas Azules -obligados en ese congreso a disolverse e integrarse en el KMT o en la YLTPP_ como de la CC Clique, que veía erosionado su control sobre las estructuras partidarias. No obstante, si sus prácticas políticas y lógica soviética en Gannan le generaron peligrosos enemigos en la élite del KMT, también dieron a CCK la palanca necesaria para seguir construyendo un espacio propio: reputación de líder incorruptible, un circulo de confianza y un discurso contra el establishment que conectaba con las aspiraciones populares, sobre todo con aquellos sectores de juventud que rechazaba la oferta política del PCCh pero que no veía esperanzas en un KMT perforado por la corrupción y las banderías.

Los antiguos Camisas Azules y CC Clique controlaban la inteligencia militar y la inteligencia política respectivamente y sometieron a CCK a una continua vigilancia con el objetivo de alertar a Chiang Kai-shek de sus simpatías comunistas. Así, por ejemplo, en verano de 1940, la inteligencia militar demostró que su entorno en Gannan estaba infiltrado por agentes del PCCh y Chiang Chingkuo fue llamado al orden por su padre. Sin embargo, los resultados de la gestión de CCK resultaban inapelables y pronto pasó a desempeñar distintas misiones de confianza (Xinjiang, Moscú, Shanghái...) para Chiang Kai-Shek así como a convertirse en el principal asesor para asuntos soviéticos. Por otro lado, la visión de CCK sobre el papel que la Liga Juvenil en la renovación del proyecto modernizador del KMT, lo llevó a convertirse con el respaldo de CKS -pero también de otros altos oficiales del KMT como por ejemplo el general Chen Cheng- primero en el director de la Escuela de Cuadros de la YLTPP y posteriormente en

36. Se conoce como "periodo de Gannan" al tiempo que CCK pasó en la hoy ciudadprefectura de Ganzhou (赣州). Esta denominación procede de la unión de dos caracteres. De una parte, la abreviatura de la provincia de Jiangxi 赣 (Gan) y de otra, el que hace referencia a sur 南 (nan). Por tanto Gannan (赣南) significaría "el sur de Jiangxi. Durante este periodo, CCK mantuvo una relación extramarital con Chang Yua-juo de la que tendría dos hijos gemelos en 1942. Poco tiempo después, en agosto, Chang murió en extrañas circunstancias. Se especula que resultó asesinada por los Servicios de Seguridad del KMT para proteger la carrera de CCK pero sin que este lo supiera. 
el responsable del departamento político del Ejercito de la Juventud, una nueva fuerza militar calificada por el propio CKS de "vanguardia revolucionaria" ${ }^{37}$. En 1945, con el rango de teniente general del Ejercito de la Juventud, CCK controlaba unos efectivos de 500.000 jóvenes.

El ascenso de CCK fue atentamente seguido por sus rivales directos en el entorno de su padre -principalmente los hermanos Chen y la familia Soong ${ }^{38}-y$ sus reveses aprovechados para erosionar su visibilidad pública. Efectivamente, a mediados de 1945, el progreso de Chiang Ching-kuo en las estructuras republicanas parecía imparable. El ámbito de la educación política de la juventud -y con él el relevo generacional de la vieja guardia- se encontraba bajo su mando; además, en el VI Congreso del KMT (1945), había logrado que la Liga adquiriese el estatus de organización del Estado encargada de la juventud separándola totalmente del Partido y finalmente se había convertido en el principal arquitecto de la política china en sus negociaciones con la URSS. Los dos Chiang consideraban que una estrategia que mezclase concesiones con firmeza podría lograr una solución de compromiso entre Moscú y Nankín tanto en lo relativo a la ocupación de Manchuria como al futuro del PCCh. Sin embargo, la ambigua posición soviética alimentada por las constantes movilizaciones anticomunistas, organizadas por los sectores más reaccionarios del KMT, dieron al traste con toda posibilidad de acuerdo. A principios de 1946, la posición defendida por CCK se consideró fallida obligándolo a adoptar un perfil más bajo en el futuro. La integración de la Liga en el KMT, adoptada por el Comité Central en 1947, supuso una nueva victoria de los sectores conservadores del Partido frente a las dinámicas más renovadoras existentes en la Liga y lideradas por CCK.

En un clima de abierta Guerra Civil entre comunistas y nacionalistas, Chiang Ching-kuo entendió nuevamente el equilibrio de fuerzas dentro del KMT y, con independencia de cual fuera su opinión, decidió no desafiar la corriente mayoritaria que, en último término, estaba liderada por su padre. En agosto de 1948, aceptó el encargo de viajar a Shanghái con el objetivo de aliviar la difícil situación económica que vivía la ciudad. CCK desplegó un ambicioso plan contra el acaparamiento, la especulación, el hampa y la fuga de capitales. En septiembre,

37. Taylor, The Generalissimo's Son..., p. 120.

38. Fruto del matrimonio entre Charlie Soong, un misionero convertido en próspero hombre de negocios, y Ni Kwei-Tseng, la familia Soong fue una de las más importantes de la joven República de China con conexiones en el mundo de los negocios, la política y las sociedades secretas. Sus tres hijas, conocidas como las Hermanas Soong (Ai-ling, Ching-ling y Mei-Ling) se casaron respectivamente con el hombre más rico de China, ministro de finanzas y descendiente de Confucio (Kung Hsiang-hsi), el fundador de la República de China (Sun Yat-sen) y el hombre fuerte de la República entre 1927 y 1975 (Chiang Kai-shek). Entre sus hijos masculinos, quizás el más conocido es Soong Tse-ven por su prominente papel político, aunque también es destacable el papel de Soong Tse-liang y sus conexiones con la banca y las grandes corporaciones. Sin embargo, el menor de los hermanos-Soong Tse-an- tuvo un perfil más gerencial y menos conectado con la política. Por tanto, Soong Mei-ling era la madrastra de CCK. 
el equipo de Chiang Ching-kuo creía que su administración podría ser un ejemplo de regeneración para todo el país, un segundo Gannan. Era un espejismo. A finales de octubre, cuando sus operaciones amenazaban con dañar seriamente los intereses de las grandes familias -entre ellas la familia Soong- los hilos se movieron en el gobierno central y fue Ilamado a Nankín por su padre ${ }^{39}$. En los siguientes meses y hasta la retirada a Taiwán, Chiang Ching-kuo acompañaría a su padre en sus diferentes desplazamientos por China hasta la derrota final. Ocupándose de aquellas tareas que le fueran encargadas -lo mismo evacuaba el tesoro nacional a Taiwán que daba moral a las tropas o las comandaba en su retirada- tardaría mucho tiempo en regresar a la primera línea de la política. Sin embargo, al convertirse en un actor secundario durante el periodo 1946-1949 minimizó el impacto en su figura de las desastrosas decisiones tomadas por la dirigencia del KMT durante la segunda fase de la Guerra Civil China.

\section{La República de China en Taiwán: de territorio defensivo a "Estado Conten- diente" $\left.{ }^{\prime 1949-1978)}\right)^{40}$}

Aunque nominalmente encargado de coordinar las operaciones de inteligencia en Taiwán desde 1949, con el objetivo de asegurar la retirada del KMT, la apretada agenda de Chiang Ching-kuo en el continente dejaron la dirección de las operaciones en manos de otros miembros del KMT, entre los que destacan Mao Renfeng -por ser en gran medida el diseñador del "terror blanco"- y Peng Mengqi, por la represión en masa llevada a cabo en Kaohsiung. Hasta mediados de 1950, CCK no tomó el control efectivo de la seguridad interna aunque, de hecho -como director de la Oficina de Información de la Secretaría Presidencial-controlaba el acceso de toda la información -tanto de la seguridad interna

39. Aunque por limitaciones de espacio no entraremos en ello, es importante señalar que las medidas de CCK en Shanghái tampoco se caracterizaron por tener fundamentos económicos sólidos conducentes a erradicar la inflación.

40. El modelo de estado fuerte que se desarrolló en Taiwán durante la segunda mitad del siglo XX no se puede entender sin la tradición materialista que existía en el seno del KMT. Este enfoque fue fruto de dos dinámicas geopolíticas. Por un lado, el KMT estableció lazos de cooperación con la Unión Soviética durante las primeras décadas del siglo XX, por el otro, el nacimiento del KMT fue producto de la voluntad política de no sucumbir ante las potencias imperialistas. Con este bagaje, el sector victorioso de las luchas intestinas desarrolló una organización partido-estado para llevar a cabo una "revolución desde arriba" con la finalidad de liberar a China de la subsidiaridad con respecto al imperialismo exterior y devolverla a su lugar entre las grandes naciones. Esta manera de concebir el Estado se trasladó a Taiwán al terminar la Guerra Civil y fue un elemento fundamental que contribuyó al exitoso desarrollo económico de la isla. Sin embargo, históricamente, el PDP ha vinculado estos aspectos materialistas de las políticas del KMT con aspectos propios del autoritarismo con el objetivo de promover un Taiwán liberal y globalista. Por consiguiente, esta interpretación fomenta una ruptura con el pasado ya que desvincula las políticas materialistas del KMT del "milagro económico taiwanés" a la vez que recoloca a Taiwán en la orbita del mundo liberal euroamericano. 
como la agencia de seguridad militar- que llegaba a Chiang Kai-shek. Las rivalidades con Mao Renfeng hombre cercano a la familia Soong- generaron un pulso en el interior de los servicios secretos de la República de China que solo concluiría cuando, la profunda reforma de los servicios de inteligencia, llevada a cabo por CCK, relegó a Mao Renfeng a una posición honorífica pero sin poder real ${ }^{41}$.

Después de la llegada del KMT a la isla de Taiwán tras la derrota en la Guerra Civil China, la seguridad interna del país se convirtió en una prioridad. El gobierno nacionalista -acosado por los comunistas, tanto fuera como dentro de la isla, la definitiva apuesta soviética por el PCCh y la falta de decisión en la Casa Blanca- necesitaba un moderno aparato de inteligencia y seguridad interna para estabilizar el nuevo orden político del KMT en el exilio. A mediados de la década de los cincuenta, los distintos servicios de seguridad, hasta la fecha dependientes del Partido o el Ejército fueron reorganizados y convertidos -al menos formalmente- en órganos del Estado.

CCK reestructuró y fusionó diversas agencias que existían en Taiwán para asegurar la estabilidad política. Como sostiene Greitens, una de las claves de esta maniobra fue la voluntad de minimizar el conflicto institucional ${ }^{42}$. El orden político no sólo dependía de la disciplina impuesta en la sociedad sino también en aquella que podía ser realizada internamente. Desde la perspectiva de CCK, el KMT no podía permitirse la debilidad interna al que los enfrentamientos de las distintas facciones lo habían sometido en el continente. Por otra parte, tal y como apunta Leng, "Chiang se benefició de hegemonizar los servicios secretos para combatir a ciertas figuras políticas con una gran influencia en los altos círculos de poder en Taiwán"43. Durante la década de los años 1950, CCK pudo asentar su posición dentro de los aparatos internos de inteligencia y seguridad. El balance de poder mundial tras la Guerra de Corea (1953) impulsó un giro diplomático de Estados Unidos hacia Taiwán que apuntaló a Chiang Ching-kuo en el juego de equilibrios dentro del KMT. Según Manthorpe, "Chiang estableció una relación de trabajo muy cercana con la CIA que le ayudó más tarde en su vida. En un punto, hubieron más de seiscientos agentes de la CIA destinados en Taiwán"44. La complicidad de Estados Unidos con Taiwán durante la Guerra Fría legitimó la represión que sufrió la sociedad taiwanesa durante la Ley Marcial (1949-1987).

41. A modo de aproximación a este punto ver: Wei, H., “蔣經國對毛人鳳的最後一擊 $\mathrm{El}$ último golpe de Chiang Ching-kuo a Mao Renfeng [Traducción propia]". NEWSSC.org (四川新 新闻网), http://www.chinanews.com/tw/lscq/news/2007/09-04/1018588.shtml (último acceso: 20/07/2020).

42. Greitens, S. C., Dictators and Their Secret Police: Coercive Institutions and State Violence. Cambridge 2016, p. 93.

43. Leng, S., Chiang Ching-Kuo's Leadership in the Development of the Republic of China on Taiwan. Lanham 1993, p. 12.

44. Manthorpe, J., Forbidden Nation: A History of Taiwan. New York 2005, p. 207. 
Efectivamente, Chiang Ching-kuo desde mediados de los años cincuenta estuvo involucrado intensamente en el denominado Terror Blanco ${ }^{45}$. Aunque partidario de una política de apaciguamiento y reeducación, las detenciones, condenas y condenas a muerte -entre quienes no cooperaban o no se retractaban de sus posiciones- continuaron durante las siguientes décadas amparadas en la Ley Marcial.

Existe un amplio debate sobre los orígenes del "Terror Blanco". Para algunos supervivientes como Chen Mingzhong, el origen está en la Guerra Civil y la lucha entre comunistas y nacionalistas, señalando que el movimiento en pro de la independencia ha distorsionado la memoria ${ }^{46}$. La narrativa actualmente dominante en Taiwán tiende a puntualizar, como indica Rigger ${ }^{47}$ que el objetivo del "Terror Blanco" fue la supresión de cualquier idea que entretuviera la posibilidad de buscar un destino independiente de China. Con independencia de donde situemos nuestro punto de vista, parece evidente que tanto el sistema de represión como el de redención estaban dirigidos a radicar cualquier crítica con el liderazgo de Chiang Kai-shek y el KMT.

La isla de Taiwán se había convertido en un puesto avanzado frente al comunismo y al mismo tiempo en una "caja de las esencias" de la civilización china, especialmente durante la Revolución Cultural en la República Popular. Los servicios de seguridad vigilaban con rigor la disidencia y la sociedad taiwanesa fue sometida a un intenso programa de sinización ${ }^{48}$. Había que estar listo para recuperar el continente, ahora robado, de las garras de los "ladrones comunistas". Un clima de movilización bélica que, paradojas de la historia, fue el inicio de un largo periodo de paz y estabilidad donde se establecieron los cimientos de un nuevo Estado taiwanés.

45. El Terror Blanco (白色恐怖) en Taiwán atravesó por distintas fases. En una primera etapa, en el contexto de la Guerra Civil y tras el Incidente de 228, la represión -en ocasiones de forma masiva - tuvo como principal objetivo personas nacidas en Taiwán. Sin embargo, a principios de los años cincuenta, y coincidiendo con la toma de control de los servicios secretos - directa o indirectamente- por parte de CCK, la represión alcanzó un carácter más político, sin distinción de origen y afectando también al ala moderada y progresista del KMT. Aunque los casos contra la disidencia política se concentraron sobre todo en la década de los cincuenta, la Guarnición de Taiwán, al amparo de la Ley Marcial realizó detenciones arbitrarias hasta el final de la década de los ochenta. Una aproximación al sistema de represión durante el Terror Blanco en Taiwán en Herrera-Feligreras A. y Lu, Y., "Espacios punitivos, entre el olvido y la memoria. El caso del Penal de San Cristóbal y el Centro de Detención de Jing-Mei", Oliver, P., y Urda, J. C., La prisión y las instituciones punitivas en la investigación histórica. Cuenca 2014, pp. 885-902.

46. Chen, M., “228被扭曲的歷史集體記憶 Incidente del 28 de Febrero: una memoria colectiva histórica distorsionada [Traducción propia]". Straits Review Monthly 172 (2005), https:// www.haixia-info.com/articles/4234.html (último acceso: 20/07/2020).

47. Rigger, S., Why Taiwan Matters: Small Island, Global Powerhouse. Lanham 2011.

48. Sobre la construcción de la identidad del Estado chino en Taiwán ver: Lu, Y., Taiwán. Historia Política e Identidad. Barcelona 2010, pp. 105-126. 


\subsection{La construcción del Estado ${ }^{49}$}

Un sector del Kuomintang llegó a Taiwán con la lección bien aprendida con respecto al potencial revolucionario del campesinado y en abril de 1949, pocos meses después de asumir su cargo como gobernador provincial, Chen Cheng empezó a impulsar las primeras medidas dirigidas a la reforma agraria. Su primer plan, denominado "reducción del arriendo 375"50, desató la ira de las élites taiwanesas que protestaron tanto en la Asamblea provincial como en el organismo encargado de la reubicación de la República de China en Taiwán ${ }^{51}$. Pero además, Chen Cheng era consciente de la desconfianza que en la población taiwanesa había despertado el Incidente de 228, por lo que trazó un programa para permitir las elecciones a nivel local como fórmula de expresión política de los isleños.

La relación entre CCK y Chen Cheng fue, en líneas generales, de cooperación aunque no exenta de rivalidades y choques de competencias en las estructuras gubernamentales. Formalmente, las tensiones entre ambos colaboradores de Chiang Kai-shek Ilegaron a su fin al crearse en 1957 el cargo de subdirector del KMT para Chang y quedando así como sucesor del viejo Chiang tanto en el gobierno como en el Partido. Sin embargo, para los observadores estaba claro que Chiang Ching-kuo era el hombre fuerte del régimen ${ }^{52}$. CCK tenía en sus manos tres resortes estratégicos para el desarrollo y estabilidad del Estado chino en Taiwán: el aparato de inteligencia y guerra política, la educación de la juventud y la relación con los veteranos ${ }^{53}$. Además, su carácter afable y cercano lo lleva-

49. El proyecto modernizador que desarrolló el KMT en la isla y la subsecuente construcción del Estado taiwanés estuvieron marcados por la combinación del desarrollo político local, la geopolítica de la Guerra Fría y las contingencias históricas a las que tuvieron que hacer frente la élite del partido nacionalista. Un proyecto que hunde sus raíces en el ideado una vez por el KMT para China pero que no fue fruto de una planificación racional tras el abandono de continente. Aunque por razones de espacio este artículo no aborda la cuestión, es importante constatar que ese "proyecto" se materializó en una realidad histórica muy especifica donde convergieron el legado materialista del KMT, las lecciones aprendidas por el KMT en los años previos a su llegada a la isla en 1949, la relación de Taiwán con Estados Unidos durante la Guerra Fría y la constatación por parte de la élite del KMT durante los años cincuenta y setenta de que la vuelta al continente era una misión imposible de realizar. Como veremos a continuación, CCK intuyó estos procesos históricos.

50. Por el que los arrendatarios no debían pagar más del 37,5\% de su cosecha anual. Antes esta medida, la renta era superior al $50 \%$.

51. 臺灣省參議會與中華民國政府遷臺

52. Central Intelligence Agency (CIA), Biographic Sketch Chiang Ching-Kuo (sic) (1 de enero de 1962), https://www.cia.gov/library/readingroom/document/0000608234 (último acceso: 15/07/2020).

53. Además de controlar de forma efectiva las distintas agencias de inteligencia y de guerra política, estaban bajo el mandato la organización juvenil China Youth Corps (中國青年救國 團) y la agencia pública relacionada con los asuntos de los veteranos denominada: Comité de Asesoramiento para Oficiales y Soldados_Retirados del Ejército Nacional (國軍退除役官兵輔 
ban a viajar -muchas veces sin escolta y por sorpresa- para tomar el pulso a la realidad de la isla. Como sostiene Leng, sus visitas al mundo agrario supusieron un punto de inflexión para Chiang $^{54}$. CCK entendió la importancia de educar a expertos que pudieran Ilevar a cabo el desarrollo económico que las áreas rurales necesitaban. Este paso reforzaría la profunda transformación económica que luego Ilegaría a Taiwán durante la década de los años 1960.

Gracias a la reforma agraria, Taiwán se benefició política y económicamente. Riger indica que por un lado, en el ámbito político, el KMT dejó de ser percibido como una fuerza extranjera y favoreció su apoyo entre los campesinos ${ }^{55}$. La implementación de la reforma cimentaba una hegemonía política que el KMT, enredado en sus compromisos con los terratenientes, no pudo construir en el continente durante los años treinta y que fue uno de los elementos cruciales de su derrota ante el Partido Comunista ${ }^{56}$. En lo económico, la reforma incrementó la productividad agraria que facilitó el desarrollo industrial que catapultó la economía de Taiwán. El impulso dado a la reforma por CCK entre los bastidores del Palacio Presidencial lo involucró, en cierta manera, en el desarrollo del proceso de acumulación primitiva que Taiwán necesitaba para competir geopolíticamente con la China de Mao.

El éxito de la reforma agraria no solo fue posible gracias a la destreza política de la familia Chiang y sus aliados más capaces como Chen Cheng o de tecnócratas como Yin Zhongrong, sino también gracias a la ayuda técnica de Estados Unidos. Según la visión geopolítica estadounidense, la ayuda a Taiwán era necesaria para evitar la expansión del comunismo en Asia Oriental después de la Guerra de Corea. En este sentido, se entiende la participación de asesores estadounidenses en la formulación políticas estratégicas tales como la reforma agraria, el desarrollo de infraestructuras o la política industrial ${ }^{57}$. Es importante destacar que la política exterior americana que acabó promoviendo una tradición estadista y desarrollista para los estados capitalistas de Asia Oriental entraba, según Golub, en contradicción con el liberalismo político hegemónico que los mismos Estados Unidos preconizaban en otros lugares del mundo. Es decir, para combatir el comunismo hacía falta una "mano visible", es decir el Estado tenía la primacía sobre el mercado ${ }^{58}$. Curiosamente, este elemento no entraba

\footnotetext{
導委員會). A mediados de los años cincuenta, los informes de la CIA Ilaman la atención sobre cómo CCK coloca a personas de su confianza en puestos clave y controla el adoctrinamiento de los oficiales. Un ejemplo en: Central Intelligence Agency (CIA), Current Intelligence BuIletin-1955/09/21 (21 de septiembre de 1955), p. 5, https://www.cia.gov/library/readingroom/ document/03000955 (último acceso: 15/07/2020).

54. Leng, Chiang Ching-Kuo's..., p. 12.

55. Rigger, Why Taiwan..., p. 47.

56. Gray, K., "Taiwan and the geopolitics of late development". The Pacific Review 24 (2011), p. 584.

57. Manthorpe, Forbidden Nation..., p. 203.

58. Golub, P., East Asia's Reemergence. Cambridge 2016, p. 59.
} 
en contradicción con la visión política del KMT, y mucho menos para $\mathrm{CCK}^{59}$. En realidad, lo que suponía un posible reto para la política exterior estadounidense en Asia Oriental se convertía en una reafirmación para el proyecto del KMT que aún estaba influenciado por el legado leninista del partido ${ }^{60}$.

De una manera mucho más directa, y visible, Chiang se implicó en el proyecto de desarrollo y construcción de infraestructuras. Este periodo (1956-1960) es un buen ejemplo del rol multidisciplinar que jugó CCK en el gobierno del KMT. Chiang Ching-kuo se involucró personalmente liderando la movilización de 10.000 veteranos, siguió de cerca el duro desafío de construcción de la Autopista Central -un proyecto estratégico desde la perspectiva militar y de desarrollo económico de la isla- que involucró también ayuda estadounidense y, al mismo tiempo -en 1958- voló a Kinmen para encargarse de la defensa de la isla, sometida a constantes bombardeos desde el continente.

Durante la década de los sesenta se produjo un paulatino relevo generacional en los órganos del Estado y en la dirección colegiada que regía las decisiones dentro del KMT. En 1965, Chiang Ching-kuo salía de la oscura comodidad tecnócrata para regresar a la primera línea política como ministro de Defensa. En el marco de la Guerra Fría, este ministerio lo situaba en una posición privilegiada en las estratégicas -y vitales- relaciones con Estados Unidos. Fortalecido -todavía más- en su posición interna, se convirtió en uno de los hombres de Washington en Asia Oriental.

\subsection{De cabeza de puente a trinchera anticomunista}

La situación geopolítica en Asia Oriental durante la Guerra Fría ofrecía un delicado balance resultado de la competición entre Estados Unidos (y sus aliados), China y la Unión Soviética. La prioridad estratégica de Estados Unidos en la región fue la contención del comunismo en pleno proceso de expansión a través de movimientos anticoloniales y el legado de la Conferencia de Bandung. En Taiwán, esta realidad geopolítica se percibía con una gran sensación de inseguridad y, en este contexto adverso, las prioridades del KMT estaban claras: sobrevivir para poder liderar la marcha futura al continente y mejorar las estructuras socioeconómicas y militares de la isla para cumplir dicho objetivo.

59. Pero Chiang Ching-kuo no era el único que pensaba en la importancia del rol del Estado. Así por ejemplo, Li Gouding (李國鼎), considerado el padre del milagro económico taiwanés aboga por el rol del Estado en el estímulo de sectores estratégicos en lo que denomina "economía libre planificada".

60. Sobre la huella leninista en el KMT y en la República de China ver: Fields, K. J., "KMT, Inc. Party Capitalism in A Developmental State". JPRI Working Paper 47, http://www.jpri.org/ publications/workingpapers/wp47.html (último acceso: 12/07/2020) y Cheng, T., "Democratizing the Quasi-Leninist Regime in Taiwan". World Politics 41 (1989), pp. 471-499. 
Winberg Chai describió el periodo de los años cincuenta y sesenta como "la década de la supervivencia" ${ }^{61}$.

Pero tras la obligación de "reconquistar" el continente, personificada en la retórica de su padre, la posición de Chiang Ching-kuo era mucho más pragmática. Como sostiene Hickey, "...en ciertas discusiones con los americanos, este [CCK] reveló que algunos hombres clave de su generación se dieron cuenta de que pasaría un largo tiempo hasta que un régimen no comunista pudiese reestablecerse en China y que quizás esto no sucedería durante sus vidas ${ }^{\prime 62}$.

Este punto es crucial para entender la visión política de Chiang Ching-kuo. Por un lado, y como se ha visto, durante toda su carrera política manifestó una gran capacidad para entender el funcionamiento de la vida política y sus complejos matices y contradicciones. Ese pragmatismo, que lo acompañó desde sus tiempos en Moscú cuando renunció a sus vínculos con la oposición trotskista, reflejaba una profunda comprensión de lo que era políticamente viable. CCK demostró, en distintas coyunturas, ser un hábil conocedor del "arte de lo posible". Por otro lado, como consecuencia de ese pragmatismo, su obra durante los primeros años en el ministerio de Defensa se centró en construir una estructura política que, en ocasiones, entraba en contradicción con la agenda pública de su padre. Dicho de otra manera, en el campo de la política exterior taiwanesa y de defensa, Chiang Ching-kuo no actuaba como si el objetivo final fuera la migración hacía otra tierra sino, más bien, como si se hubiera de anclar el proyecto político del KMT a la geografía en la que el destino les había abocado.

Lo que estaba claro era que para cumplir sus objetivos, Taiwán necesitaba de Estados Unidos y su paraguas de seguridad ${ }^{63}$. Desde su primera visita a EE. UU. en 1953, CCK tuvo un rol central a la hora de tejer las relaciones entre el gobierno chino en Taiwán y Estados Unidos. Durante la década de los años sesenta, desarrolló vínculos estrechos con la comunidad de inteligencia estadounidense estacionada en la isla. Según Taylor, Chiang era "el principal negociador entre bambalinas con Estados Unidos sobre asuntos internacionales delicados" ${ }^{164}$. Tras su llegada al ministerio de Defensa este horizonte de colaboración se ensanchó

61. Hsiung, J., "Diplomacy against Adversity: Foreign Relations under Chiang Ching-kuo". Asian Affairs: An American Review 27 (2000), p. 111.

62. Hickey, D. V. H., Foreign Policy Making in Taiwan. From Principle to Pragmatism. London 2007, p. 85.

63. Algo similar a lo que ocurría en España. Ambas dictaduras estaban integradas en el sistema estratégico estadounidense. Para una aproximación a las relaciones entre España y la República de China: Herrera-Feligreras, A., España y China (1973-2005). Del reconocimiento diplomático a la Alianza Estratégica. Barcelona 2015, pp. 43-48; Para profundizar en lazos entre Madrid y Taipéi como "trincheras anticomunistas" en Europa y Asia ver: Del Río MoriIlas, M.A., "La conexión anticomunista sino-española: Chiang Kai-shek y Franco (1953-1973)". Dictatorships \& Democracies. Jounal of History and Culture 5 (2017), pp. 249-281, https://doi. org/10.7238/dd.v0i5.3139.

64. Hickey, Foreign Policy..., p. 83. 
en el teatro asiático ampliando la colaboración con la Agencia Central de Inteligencia (CIA) en Taiwán y usando la isla como base para desarrollar operaciones de guerrilla y espionaje en China y Birmania. Wang Sheng, leal colaborador de Chiang desde los años treinta, se convirtió en el referente en el área de las operaciones anticomunistas ${ }^{65}$. Ya en 1961 estaba exportando el modelo de guerra política de la República de China a Vietnam; la entrada de Estados Unidos en este conflicto (1964) no hizo sino estrechar los lazos personales de CCK con el gobierno estadounidense ${ }^{66}$ y poner en valor la posición de Taiwán: en 1966, Taipéi se convertía en sede de la Liga Anticomunista Mundial.

La dependencia de Estados Unidos no impidió al circulo de poder del KMT buscar ampliar su margen de autonomía. Así por ejemplo, la República de China mantuvo contactos secretos con la Unión Soviética -políticamente intensos entre 1963 y 1971- buscando algún tipo de entendimiento estratégico respecto a la República Popular. Sin embargo, la posición de liderazgo moral de la URSS en el campo socialista, el miedo de la élite del KMT a poner en peligro los lazos con Washington y una posible intervención de la República Popular limitaron la cooperación entre Moscú y Taipéi ${ }^{67}$.En el área de la defensa nacional, Chiang Chingkuo revolucionó la industria militar. En 1965 estableció el Instituto Nacional de Ciencia y Tecnología Chung-Shan (NCSIST). En opinión de Liu, el objetivo de CCK consistía en "fomentar la industria de defensa local para evitar una excesiva dependencia de ayuda militar externa"68. El establecimiento de una industria militar local anclaba aún más al KMT a la isla. Más allá del apoyo a Estados Unidos en su cruzada anticomunista en Asia Oriental, la visión geoestratégica, en lo militar, de CCK era mucho más pasiva que activa. Taiwán dejaba de ser una cabeza de puente provisional, desde la que lanzar la ofensiva hacia el continente, y el objetivo pasó a ser defender lo conseguido a través de una estrategia de hechos consumados. Una estrategia que Chiang Ching-kuo ensancharía, durante su etapa como Primer Ministro, a otros ámbitos de la gobernanza.

65. Ver Lin, H., "Taiwan's Cold War in Southeast Asia | Wilson Center". CWIHP E-Dossier 70 (2016), https://www.wilsoncenter.org/publication/taiwans-cold-war-southeast-asia (último acceso: 01/07/2020).

66. Manthorpe, Forbidden Nation..., p. 207.

67. Existe un interesante debate sobre las posibilidades, alcance y limitaciones de las relaciones entre la República de China y la URSS desde el clásico trabajo de Gaver de los años setenta: Garver, J., "Taiwan's Russian Option: Image and Reality". Asian Survey 18 (1978), pp. 751-766; Aportaciones más recientes a este debate en: Share, M., "From Ideological Foe to Uncertain Friend: Soviet Relations with Taiwan, 1943-82". Cold War History 3 (2003), pp. 1-34; Tubilewicz, C., "Taiwan and the Soviet Union During the Cold War: Enemies or Ambiguous Friends?". Cold War History 5 (2005), pp. 75-86; Zhai, X. y Xiao, R., "Shifting political calculation: the secret Taiwan-Soviet talks, 1963-1971". Cold War History 15 (2015), pp. 533-556.

68. Liu, F., "Taiwan's Security Policy Since The Cold War Era: A Review Of External Military Assistance And The Development Of Indigenous Defence Industry". History Of Global Arms Transfer 9 (2020), p. 9. 


\subsection{Años de estrés}

Durante los años setenta, la salud de Chiang Kai-shek se deterioró considerablemente y Chiang Ching-kuo fue nombrado Primer Ministro de la República de China en 1972. El período de su mandato como Premier (1972-1978) ha sido descrito por Clough como una etapa de "estrés severo"69. CCK era consciente de la difícil situación que atravesaba Taiwán. Por un lado, la delicada salud de su padre avanzaba el fin de una figura simbólica (moriría en 1975) y amenazaba la legitimidad histórica del proyecto del KMT como partido guía de la modernización de China. Por otro, en Estados Unidos soplaban vientos de cambio, algo que el propio Chiang había percibido durante su última visita a Washington en $1970^{70}$. El respaldo del KMT a la Doctrina Nixon y su activo papel de soporte a los regímenes anticomunistas del área parecían poca cosa frente a la voluntad de Nixon y Kissinger de establecer relaciones diplomáticas con la República Popular China. Un año más tarde, en 1971, la expulsión de la República de China de la Asamblea General de las Naciones Unidas y la consecuente admisión de la República Popular China confirmaron los peores pronósticos en el círculo de poder del Kuomintang. El dominó de pérdida de asientos en organismos multilaterales y de reconocimiento internacional por parte de otros Estados, abrió una crisis de legitimidad que solo podría afrontarse coexistiendo con el cambio de enfoque de los aliados, articulando una oposición anticomunista en el área que sirviese a sus objetivos de seguridad nacional y, sobre todo, impulsando el desarrollo económico de la isla.

Los reveses diplomáticos sufridos por el Gobierno de la República de China en Taiwán, a partir de su salida de Naciones Unidas en 1971, tuvo una repercusión directa en la estabilidad económica de la isla reduciendo la inversión privada, provocando -a través de una ola de migraciones masivas- una grave salida de capital al extranjero ${ }^{71}$ y, finalmente, la crisis del petróleo impactó a una economía dependiente del exterior. Sin embargo, ese panorama adverso, facilitó la acción política de los sectores más reformistas del KMT despertando a la vieja guardia del mito de la "reconquista del continente". Chiang Chingkuo y el grupo de tecnócratas que dirigían la política taiwanesa, dejaron de tomar Taiwán como una plataforma provisional al tiempo que aumentaban su margen de maniobra para impulsar una política que cimentara la legitimidad interior $^{72}$. A pesar de la crisis diplomática, su pragmatismo político lo llevó

69. Hsiung, "Diplomacy against..., p. 112.

70. Durante esta visita a EE.UU, el 24 abril de 1970, CCK fue objeto de un intento de asesinato por varios miembros de la World United Formosans for Independence (WUCI), una organización fundada en 1970 cuyo objetivo es establecer la República de Taiwán.

71. Lee, K., 蔣經國與後蔣時代的內閣政治菑英 (1972 1993年) Elites políticas del gabinete en la era post Chiang Ching-kuo (1972-1993) [Traducción propia]. Taipei 2014, pp. 47-48.

72. Kapur, H., Taiwan in a Changing World: Search for Security. Bloomington 2004, p. 22. 
a trabajar con los mimbres que tenía en su mano lo que seguía implicando mantener -a pesar del giro político de la Casa Blanca- una excelente relación con Estados Unidos.

Como ya se ha hecho referencia, el legado leninista del KMT aún seguía fundamentando sus decisiones a pesar de la tradición y retórica anticomunista del Partido. Una prueba de ello es el papel que la planificación económica jugó en esta fase de desarrollo de la economía de Taiwán y que se concretó en iniciativas, bajo el gobierno de CCK, como la de los "Diez Principales Proyectos de Construcción (1974-1979)"173. Este plan, de 300 millones de dólares taiwaneses, incluía seis proyectos relacionados con el transporte, tres con el sector industrial y la construcción de una central eléctrica. Es importante destacar que, a pesar de la primacía del Estado a la hora de liderar dicho proceso económico, la modernización del país también fue posible gracias a la cooperación con empresas privadas y el continuo apoyo estadounidense que facilitó la acumulación de capital en un entorno más o menos securitizado. En este sentido, si Li Guoding es el padre económico del "milagro económico taiwanés", Chiang Ching-kuo es su padre político. El trabajo iniciado durante la década de los cincuenta y la movilización de recursos estatales durante las décadas posteriores fueron clave para alcanzar ese alto nivel económico. También es necesario destacar, una vez más, ese pragmatismo con ideas propias que le caracterizaban en la toma de decisiones. A pesar de que era consciente de que el liberalismo económico era la doctrina hegemónica en Estados Unidos, y otras regiones del mundo tuteladas por la potencia estadounidense, CCK no dudó en apadrinar una doctrina económica que, en ocasiones, entraba en contradicción la doctrina de sus mecenas ${ }^{74}$.

Con el triunfo de su viejo camarada de los años de Moscú -Deng Xiaopingen la lucha de poder abierta en el PCCh tras la muerte de Mao, y tras una década de aproximaciones, la Administración Carter normalizaba las relaciones con Pekín. Chiang Ching-kuo Ilevaba poco más ocho meses como presidente de la República de China y ahora, más que nunca la localización del Partido, el Estado y la Economía se convertía en un asunto vital.

\section{La República de China en Taiwán busca su sitio (1978-1988)}

Efectivamente, a las dos de la madrugada del 16 de diciembre de 1978, Chiang Ching-kuo fue informado de que Washington y Pekín habían anunciado que establecerían relaciones diplomáticas plenas el 1 de enero. Consecuentemente romperían con Taipéi y pondrían fin al tratado de defensa firmado en

73. 十大建設

74. La necesidad de una nueva legitimidad fue una idea compartida por un grupo de dirigentes del KMT a los que CCK les dio el necesario apoyo político como hombre fuerte del régimen. Peng, H., 台灣政治發展 Political development in Taiwan. Taipei 2003, p. 283. 
$1954^{75}$. Por su parte, la dirigencia de la República Popular China, emitió -el 1 de enero de 1979- su quinto "Mensaje a los compatriotas en Taiwán", en el que sustituyó el reiterado Ilamado a "liberar Taiwán" por "solucionar pacíficamente el problema de Taiwán", reforzando activamente la idea de una "reunificación

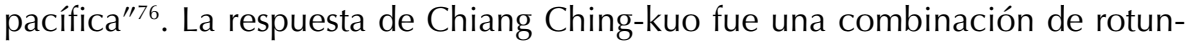
didad -con la declaración de los "Tres Nos" (no contactos, no concesión, no negociación) y posibilismo, desarrollando una diplomacia flexible que permitiese a la ROC mantener relaciones de facto con países sin reconocimiento diplomático y las organismos multilaterales así como aceptando la denominación de Chinese Taipei para asistir a eventos internacionales.

La ofensiva diplomática de Deng en pro del entendimiento entre ambas oriIlas tuvo en la fórmula de "un país, dos sistemas" su principal fundamento. La respuesta de su viejo amigo desde Taipéi fue que "Un país necesita solo un buen sistema"77. En cualquier caso, en este ambiente de deshielo, el desarrollo económico de Taiwán y la política de Reforma y Apertura promovida por Pekín, desbordaron las posiciones políticas iniciándose un movimiento comercial y de inversión en el que Hong Kong hacia el papel de puente hacia el inmenso mercado de la República Popular sin ninguna cortapisa por parte del gobierno del CCK quien, sin embargo, a pesar de las presiones de distintos sectores sociales, no autorizaría los contactos y visitas al continente hasta $1987^{78}$.

\section{1. Éxito económico y localización política}

Tras su toma de posesión como presidente de la República de China, Chiang Ching-kuo profundizó, a través de sucesivos planes de desarrollo económico, en su política de construcción de infraestructuras, modernización de la agricultura e impulso de las industrias estratégicas. A finales de 1980, se estableció el parque científico e industrial de Hsinchu, símbolo de una estrategia que acaba-

75. Desde entonces, las relaciones entre la República de China (Taiwán) y Estados Unidos se canalizan a través del Instituto Americano en Taiwan (American Institute in Taiwan) y el marco de seguridad está estipulado por la Taiwan Relation Act (TRA).

76. El mensaje dirigido a Taiwán en enero de 1979 constituye el pistoletazo de salida de esta nueva política articulada desde China modificando, sustancialmente, su estrategia hacia Taiwán: Standing Committee of the Fifth National People's Congress, Message to Compatriots in Taiwan, 1 de enero de 1979, http://www.china.org.cn/english/7943.htm (último acceso: 03/07/2020).

77. Así lo explicó, en una entrevista a comienzos de 2019, el ex presidente Ma Yingjeou en: Shih, H., y Chung, J., "Xi Jinping Speech: Xi's position differs from the KMT's: Ma Ying-jeou". Taipei Times, 4 de enero de 2019, http://www.taipeitimes.com/News/taiwan/archives/2019/01/04/2003707321 (último acceso: 07/06/2020).

78. Sang, X., "Power, Interests, and Internal Factors. A Neoclassical Realist Perspective on the Taiwan Issue". Ghent 2017, pp. 34-35, https://biblio.ugent.be/publication/8534625/ file/8534683.pdf (último acceso: 03/06/2020). 
ría haciendo de Taiwán uno de los Cuatro Tigres Asiáticos. La promoción de la creación de empresas y el rol del gobierno, a través tanto de políticas de estímulos como de desregulación de determinados sectores, ocuparon un lugar central en la conversión de Taiwán en lugar de referencia en las cadenas de suministros globales $^{79}$ : un objetivo buscado para que el país superase sus dificultades de reconocimiento diplomático ${ }^{80}$.

Si el desarrollo económico era uno de los pilares para la nueva legitimación buscada por Chiang Ching-kuo, el otro era borrar la divisoria invisible existente en la sociedad isleña. Uno de los sutiles mecanismos de vigilancia y control de la lealtad de la población fue su clasificación según su origen provincial -bensheng ren, gente de esta provincia (Taiwan)-, y los waisheng ren, gente de fuera de esta provincia-. Esta "clasificación", terminaba por convertir en sospechoso a todo bensheng ren y reservaba, de facto, las responsabilidades políticas, económicas y militares para los waisheng ren ${ }^{81}$. Desde su nombramiento como primer ministro, CCK había impulsado la integración de los bensheng ren en el KMT a través de Lee Huan ${ }^{82}$, responsable de organización del Partido desde 1972. Sin embargo, tras el Incidente de Zhongli (1977), Lee se vio obligado a renunciar por las presiones del ala derecha del KMT.

En marzo de 1981, en el XVI Congreso de KMT, Chiang Ching-kuo afirmaba que la reunificación bajo los Tres Principios del Pueblo era el único camino viable para modernizar China. CCK consolidaba así su visión sobre como la guerra política debía sustituir cualquier pretensión de enfrentamiento militar. Una sociedad china en Taiwán moderna, y al mismo tiempo orgullosa de sus tradiciones, en un continuo crecimiento de bienestar material debía convertirse en una opción más atractiva para los compatriotas del continente ${ }^{83}$, donde todavía humeaban las cenizas de la Revolución Cultural (1966-1976).

Como en otras ocasiones, CCK esperó a que la tormenta se calmase dentro de los órganos de decisión del partido-estado para continuar con su política reformista. Una vez presidente, recuperó a Lee Huan. Primero para el ministerio de Educación (1984), donde llevó a cabo reformas tanto de profundidad como simbólicas -por ejemplo, terminando las restricciones sobre la longitud

79. Chen, H., “我國經濟自由化之探討 A Study on Economic Liberalization Policy in Taiwan". 經濟研究 Economic Research 13 (2013), pp. 183-206.

80. Hickey, Foreign Policy..., p. 85.

81. Más sobre esta "división del trabajo" en Murai, Tomoko. "La formación de la identidad taiwanesa en el siglo XX". México y la Cuenca del Pacífico 2 (1999), pp. 25-26. Huang, C., 日據 五十年統治對光復後台灣之影響 La influencia de los 50 años de la ocupación japonesa sobre Taiwán tras su retorno a China [traducción propia]. Taipei 2002, p. 44.

82. Sobre la figura de Lee Huan: Brown, K., "Lee Huan obituary. Former premier of Taiwan who played a key role in the 1970s". The Guardian, 12 de diciembre de 2010, https://www. theguardian.com/world/2010/dec/12/lee-huan-obituary (último acceso: 05/07/2020).

83. Sang, "Power..., p. 34. 
del cabello de los estudiantes-, y posteriormente como Secretario General del KMT (1987) y desde esa posición continuó abriendo canales de participación para los nativos taiwaneses en la dirección del Partido y el Estado. El porcentaje de locales en el gabinete presidencial de Chiang Ching-kuo durante su primer mandato alcanzó el 29,6\% y llegó hasta el 32,1\% en su segundo mandanto ${ }^{84}$.

La legitimación del régimen vía cooptación se intensificó y terminaría por convertir a los bensheng ren en mayoritarios en el Partido y el Ejército ${ }^{85}$. Este ascenso a la elite dirigente de los bensheng ren redundará en una paulatina "localización" de la política taiwanesa siendo este -tanto por su implicación en las estructuras de poder como por su retorno activo a otras áreas de la política- uno de los legados del mandato de Chiang Ching-kuo.

\subsection{Nuevos actores en la vida política de Taiwán}

La República de China en Taiwán se presentaba al mundo como la "China libre" en oposición a la dictadura del PCCh en el continente y el puesto más avanzado en Asia frente al autoritarismo comunista. La cronología oficial del Kuomintang señala el 25 de diciembre de 1947 como el final de la fase de "tutela política" e inicio de la fase constitucional ${ }^{86}$. Sin embargo, la realidad era que la legislación de excepción suspendía derechos constitucionales y dejaba, únicamente, el nivel local como único canal de expresión política de la población no encuadrada en el KMT. Estos últimos eran conocidos como candidatos del Dangwai (literalmente, de fuera del Partido, es decir, de fuera del Kuomintang) y pronto agruparían los movimientos de oposición.

Tanto la oposición de origen comunista o de izquierdas como la de carácter más liberal fueron convergiendo al final de la década de los sesenta en el movimiento de memoria histórica y derechos humanos. La atmósfera de relativa tolerancia durante el mandato de Chiang Ching-kuo llevó a los taiwaneses a un mayor interés por los asuntos públicos y hacer de las campañas locales y provinciales un mecanismo para expresar ideas o denunciar -dentro de unos márgenes- aspectos tales como la corrupción, fraudes o excesos del régimen.

Los candidatos del Dangwai -formado principalmente por intelectuales, profesionales y jóvenes de la élite taiwanesa aunque también candidatos cuyo origen era la clase trabajadora-, catalizaron las aspiraciones sociales y actuaron como promotores de la democracia desde la base, formando gradualmente un grupo político de oposición. Este movimiento encontró soporte financiero

84. Lee, 蔣經國..., pp. 58 y 93.

85. Kung, I.,「外來政權」與本土社會 El “régimen extranjero" y la sociedad civil taiwanesa [traducción propia]. Taipei 1998, pp. 221-222.

86. Reflejado en la cronología pública del KMT en su propia página web: http://www1. kmt.org.tw/english/page.aspx?type=para\&mnum=108 (último acceso: 01/07/2020). 
en los terratenientes perjudicados por la reforma agraria de los años cincuenta que, a partir de este periodo, regresan a la vida política de la isla y con ello el movimiento independentista comienza a bascular su centro desde el exterior al interior de la isla ${ }^{87}$.

En la segunda mitad de 1979, el Dangwai, hasta ese momento un movimiento informal, fundó la "Asociación de los Candidatos del Dangwai" y la revista Formosa o Meilidao. La inauguración de cada una de las delegaciones de la revista se convertía en un acto político pro-democracia en una dinámica que reflejaba la vibrante sociedad existente bajo la uniformidad de la Ley Marcial. El 10 de diciembre, con motivo del Día Mundial de los Derechos Humanos, se celebró en Kaohsiung una manifestación reivindicando derechos políticos y reformas democráticas en Taiwán. Aunque el encuentro contaba con los permisos para su celebración, los lemas y la actitud de los participantes fue considerada por las autoridades como una provocación y fue disuelto violentamente por las fuerzas de orden público. Dos días después, el Gobierno llevó a cabo una operación policial de gran envergadura deteniendo a una cantidad de personas relacionadas con la publicación. A todos ellos se les aplicó la legislación derivada de la Ley Marcial y fueron condenados, ejemplarmente, por un Consejo de Guerra en marzo de 1980. Esta escalada de detenciones y el posterior juicio es conocido como el Incidente de Meilidao o el Incidente de Kaohsiung.

Desde comienzos de la década de los ochenta, los juegos de poder en el interior del KMT, entre los sectores más conservadores y reformistas, continuaban al mismo tiempo que, como hemos visto, se iniciaba un pulso entre los dirigentes del partido-estado y una oposición cada día más asertiva. Al igual que había pasado con su padre, el acceso a la presidencia de la República de China ensalzó el culto a la personalidad de Chiang Ching-kuo vigilando cualquier tipo de manifestación que pudiera considerarse lesiva para su persona. En este marco se sitúa el asesinato, en suelo estadounidense, de Henry Liu ${ }^{88}$ por su intención de publicar una biografía - no autorizada- sobre CCK. El jefe de la Oficina de inteligencia Wang Hsi-ling reclutó al jefe de la Unión de Bambú - una de las mayores triadas en Taiwán que colaboraba con la seguridad del Estado- Chen Chi-li y le encargó el asesinato de Henry Liu. EI FBI resolvió muy pronto el caso, funcionarios estadounidenses acusaron al KMT de orquestar el asesinato y la relación entre Taipéi-Washington se volvió muy tensa.

Naturalmente, el gobierno de la República de China se desmarcó de este asunto y CCK reaccionó actuando en tres direcciones. En primer lugar, respondió a las presiones de los Estados Unidos procesando a los principales implica-

87. Winckler, E. A. y Greenhalgh, S. (eds.), 臺灣政治經濟學諸論辯析 Contending Approaches to the Political Economy of Taiwan. Taipei 1994, pp. 192-193.

88. Conocido bajo el seudónimo de Chiang Nan (江南; Jiāng Nán). 
dos en el asesinato de $\mathrm{Liu}^{89}$. En segundo lugar, y con el objetivo de demostrar la inexistencia de lazos entre su gobierno y el crimen organizado, en menos de un mes llevó a cabo el famoso "Proyecto de Limpieza"90, una operación desarrollada por la Guarnición de Taiwán -el brazo ejecutivo del servicio secreto- amparado en la Ley Marcial que detuvo 4.000 miembros de las triadas con sentencias de hasta 30 años. Esta acción tenía como principal objetivo detener a Chen Chi-li, sin embargo, terminó por llevar a la cárcel a los principales caudillos de las bandas mafiosas. En prisión, tomaron conciencia sobre la importancia de controlar la política y las triadas aprovecharon la paulatina apertura política para "blanquearse", postulando sus propios miembros como candidatos o cooperando con políticos esperando compartir después intereses y poder ${ }^{91}$. De esta manera, se inició una cultura de dinero negro en la política taiwanesa.

Finalmente, y aunque los informes de la CIA lo descartaban ${ }^{92}$, el escándalo provocado por el asesinato desató el rumor de la instauración de una dinastía familiar en el Gobierno de la República de China. CCK aprovechó la oportunidad para continuar con su guerra política frente a la República Popular. En un discurso ante la Asamblea Nacional en diciembre de 1985 puso fin a las especulaciones haciendo referencias indirectas al escándalo por el asesinato de Henry Liu y subrayando los mecanismos constitucionales y parlamentarios del la República de China -frente a la República Popular- en la próxima elección presidencial $^{93}$.

Incidentes como el de Zhongli (1977) o Meilidao (1979-80) contra la oposición democrática, u operaciones llevadas a cabo contra el inframundo como el "Proyecto de Limpieza" (1985), tenían como denominador común la desarticulación de todo movimiento que supusiera un potencial peligro activa o pasivamente- para la estabilidad del régimen. Sin embargo, el efecto inmediato fue un mayor interés y atención por la política. Mucha gente,

89. Como resultado de la presión de EE. UU., el 10 de enero de 1985 el jefe de la Oficina de inteligencia Wang Hsi-ling fue condenado a cadena perpetua. Sin embargo, le prepararon en el Centro de Detención de Jing-Mei un edificio individual para vivir. Salió de prisión el 21 de enero de 1991.

90. 一清專案

91. Ren, Y., “台灣地區基層民主選舉中的「黑金政治」Política de dinero negro en las elecciones democráticas a nivel local en Taiwán [traducción propia]". Twenty-First Century [Versión digital] 38 (2005), http://www.cuhk.edu.hk/ics/21c/media/online/0411019.pdf (último acceso: 28/10/2020).

92. Central Intelligence Agency (CIA), Taiwan After Chiang Ching-Kuo: A Speculative Analysis (1 de septiembre de 1982), https://www.cia.gov/library/readingroom/docs/CIA-RDP84S00554R000100140003-0.pdf (último acceso: 01/06/2020).

93. El discurso puede consultarse completo en: Chiang, C., "Chiang: Constitution To Determine His Successor: President Bars Military Rule, No Family Member To Be Elected". Free China Journal, el 30 de diciembre de 1985 [A partir de 2009, esta publicación pasó a llamarse Taiwan Today], https://web.archive.org/web/20160808114036/http://www.taiwantoday.tw/ ct.asp?xltem=117721\&CtNode=103 (último acceso: 02/06/2020). 
durante las elecciones de comienzos de los años 80, dio el voto a las nuevas candidaturas del Dangwai, formadas por familiares y abogados de los represaliados por el Incidente del Meilidao. Entre estos últimos destaca Chen Shuibian, presidente de Taiwán durante 2000-2008. Junto con la reconstrucción del movimiento del Dangwai y la recuperación de las reivindicaciones democráticas, se inició un proceso de retorno de aquellos exiliados políticos bensheng ren -tanto de izquierda como partidarios de la independencia taiwanesa- que se encontraban fuera del país desde los años 50. Estas dos corrientes -Dangwai y retornados- convergieron en la creación de un partido opositor: el 28 de septiembre de 1986 se fundó el Partido Demócrata Progresista (PDP) o el Minjindang.

En un clima internacional marcado por el inicio de la distensión entre Estados Unidos y la Unión Soviética, la política de reforma y apertura impulsada por Moscú y los primeros pasos de reconciliación entre el Kremlin y Zhongnanhai, CCK parecía tener claro que era necesario reforzar el perfil democrático del país en el teatro de las naciones. No solo no intervino en la creación del PDP, sino que declaró que el Kuomintang debía promover las reformas necesarias para adaptar Taiwán a los nuevos tiempos ${ }^{94}$.

Los paulatinos reajustes implementados, desde principios de los años ochenta, en la agenda pública del KMT permitió a Chiang Ching-kuo avanzar en su proyecto de legitimación interior frente al "bunker" dentro de su propio Partido. En julio de 1987, CCK estableció tres objetivos para Lee Huan: reformar el KMT, avanzar hacia la democracia y la reunificación ${ }^{95}$. Ese mismo mes, ordenaba el levantamiento de la Ley Marcial que duró 38 años -la más larga del mundo- abriendo la puerta a la legalización de los partidos políticos; en agosto, se iniciaron los contactos políticos - de carácter oficioso- entre las dos orillas del Estrecho y se permitió la apertura de los contactos familiares con China continental ${ }^{96}$. Esta nueva realidad, abría un futuro para el diálogo entre Taipéi y Pekín.

Dentro del Kuomintang, Lee Huan transformó los objetivos indicados por CCK en líneas políticas. En septiembre de 1987, en un discurso pronunciado en Kaohsiung, siete años después del incidente Meilidao, anunciaba que el objetivo del Kuomintang no era reemplazar al gobierno del PCCh en el continente, sino "presionar por la democracia, la libertad de prensa y una economía abierta en el continente para librar a China del comunismo y avanzar hacia un estado moderno democrático" ${ }^{\prime \prime 7}$. La reacción no se hizo esperar y el sector ultra del

94. Lee, S., 快讀台灣史 Breve historia de Taiwán [traducción propia]. Taipei 2002, p. 139.

95. Hu, C., "Taiwan's geopolitics and Chiang Ching-Kuo's decision to democratize Taiwan". Stanford Journal of East Asian Affairs 5 (2005), pp. 26-44.

96. Sang, "Power..., pp. 34-35.

97. Hu, "Taiwan's geopolitics..., p. 32. 
KMT lanzó una ofensiva contra él acusándolo de traicionar los compromisos históricos del Partido. Pero CCK lo respaldó ordenando la publicación del discurso en el órgano del $\mathrm{KMT}^{98}$.

La fundación del PDP, con la complicidad del gobierno de CCK, había sido un claro mensaje sobre el nuevo tiempo político que se inauguraba en Taiwán. A lo largo de 1987, especialmente tras el levantamiento de la Ley Marcial, comenzaron a visibilizarse toda clase de movimientos políticos, sociales y culturales $^{99}$. Anunciada en los últimos meses de 1987, el 1 de enero de 1988 entraba en vigor el levantamiento la prohibición de periódicos ${ }^{100}$ Poco después, el 13 de enero murió el Chiang Ching-kuo, abriendo la puerta a la transición política en Taiwán.

\section{Conclusión}

No es posible recorrer la vida de Chiang Ching-kuo sin hacerlo, al mismo tiempo, por los episodios críticos que conforman la historia del Mundo Chino en el siglo XX. Vínculos geopolíticos, recorridos biográficos, transformaciones sociales, económicas, etc., conforman una pléyade fruto de un esfuerzo de modernización que acabarían cristalizando en dos organizaciones opuestas: el KMT y el PCCh. Sin embargo, antes de constituirse en némesis, los rivales tuvieron un origen y propósito común y ahí esta la biografía de CCK para hablarnos de la profunda huella en la concepción y fines del Partido y el Estado, así como del papel de China en el mundo, que tanto comunistas como nacionalistas chinos comparten.

Desde su más temprana juventud, Chiang Ching-kuo demostró el propósito de contar con una agenda propia y su capacidad para adaptarse a las circunstancias. La decisión de ir a estudiar a Moscú, su apuesta por el proyecto emancipador comunista y su integración en las estructuras partidarias, estatales y profesionales soviéticas al igual que, a partir de 1937, lo estaría en las auspiciadas por el KMT lo prueban. En este contexto, ha quedado demostrado que CCK nunca fue un rehén en manos de Stalin, más bien al contrario destacó por

98. El expresidente Ma Ying-jeou señaló que cuando trabajaba para Lee Huan, ambos fueron calificados como "Guardias Rojos" debido a su trabajo reformista. Taipei Times, "Ma praises Lee Huan for role in political reforms". Taipei Times, el 20 de diciembre de 2010, http://www.taipeitimes.com/News/taiwan/archives/2010/12/20/2003491431 (último acceso: 12/07/2020).

99. Sobre las transformaciones vividas en el interior del KMT, la apertura gradual del régimen, las características del PDP y la gobernanza política en Taiwán vease los trabajos de: Tien, H., The Great Transition. Political and Social Change in the Republic of China. Stanford 1989; Rigger, S., From opposition to power. Taiwan's Democratic Progressive Party. London, 2001; Fell, D., Government and Politics in Taiwan. London 2012.

100. United Daily News 聯合報, 15.X.1987, p. 1. 
su desempeño profesional al servicio del Estado soviético. Este aspecto de la vida de CCK ha sido ya trabajado por cierta historiografía especializada que ha trabajado con fuentes soviéticas; no obstante, lo consideramos un aporte novedoso a la literatura en español sobre estudios chinos y taiwaneses.

No puede decirse que Chiang Ching-kuo fuese un gran teórico de la revolución y la construcción de la nueva China, sin embargo si parecía tener una visión del papel que el Estado y el Partido debían jugar para mejorar las condiciones de la vida de la gente común. Una visión que hunde sus raíces en su experiencia soviética y de gestión en Gannan y Shanghái que le dieron fama por su carácter sencillo, amigable, incorruptible y pragmático. Realismo, al que sometió sus ideas y opiniones personales, y que le sirvió para tomar posiciones, con gran olfato político en el seno de KMT hasta convertirse en Presidente de la República de China ${ }^{101}$. Un desenlace que, posiblemente, pocos en la dirección del partido-estado podrían imaginar cuando regresó a China en 1937.

Chiang Ching-kuo fue una de las principales mentes que contribuyó a la construcción y al desarrollo del Estado taiwanés. En este sentido, el Taiwán actual no se puede entender sin su obra. Su heterodoxia y su comprensión de la política desde un enfoque materialista fue crucial para el desarrollo económico del Estado. Si bien es cierto que la tutela de Estados Unidos juega un rol importante en el "milagro taiwanés" también es cierto que nadie en Washington fue capaz de prever el desarrollo de Taiwán como potencia económica regional bajo el mandato de $\mathrm{CCK}^{102}$. Este elemento contrasta con el discurso, ampliamente aceptado, que celebra el éxito del "milagro taiwanés" como una victoria del liberalismo económico promovido desde Washington y de su geopolítica en Asia Oriental durante la Guerra Fría. Consideramos que esta narrativa dominante elimina el legado materialista existente en el KMT, que fue un elemento articulador de la política del Partido, y que CCK maximizó en sus propuestas de gobierno. En este sentido, concluimos sugiriendo una futura línea de investigación crítica con el relato esgrimido por sectores tanto del nacionalismo taiwanés -que sobredimensionan el autoritarismo del Kuomintang-, como dentro del KMT obsesionados con distanciarse del "pecado original soviético".

En ese sentido, el "milagro taiwanés" hundiría sus raíces en el proyecto modernizador imaginado una vez para China y que - mutatis mutandis- acabó, con un protagonismo claro de CCK, viendo la luz en Taiwán. Un proyecto

101. Chiu, 蔣經國..., pp. 49-158.

102. Thomas Shoesmith, diplomático estadounidense encargado de los asuntos de la República de China entre 1966 y 1971, al reconocer que en Estados Unidos nadie había previsto la fortaleza económica desarrollada por Taiwán señala: "El sucesor de Chiang Kai-shek, Chiang Ching-kuo, no dio señales de que era un hombre con la amplitud de visión que posteriormente demostró". Kennedy, S., "AMBASSADOR THOMAS P. SHOESMITH". Studies and Training Foreign Affairs Oral History Project, 9 de diciembre de 1991, p. 18, https://www.adst. org/OH\%20TOCs/Shoesmith,\%20Thomas.toc.pdf (último acceso: 15/07/2020). 
modernizador -con reflejos en ambos lados del estrecho de Formosa- que arranca en los reformadores de la última etapa de la dinastía Qing y va tomando cuerpo a lo largo de un corto siglo XX chino. Un siglo que se inicia con el nacimiento de la República en 1912 y que a comienzos de la década de los noventa verá como ese universo civilizatorio que Ilamamos China entra en una nueva etapa.

La muerte repentina de Chiang Ching-kuo abrió una crisis que quizás, con el nombramiento de Lee Teng-hui como sucesor, se cerró de forma inesperada. CCK, dejo un final abierto a múltiples interpretaciones que facilitó el triunfo de los reformistas en el KMT y la senda hacia la transición democrática. Tal vez por ello, a pesar de sus implicaciones en el Terror Blanco, es hoy el presidente con mayores índices de popularidad de la historia de Taiwán ${ }^{103}$.

\section{Bibliografía}

Banac, I., The Diary of Georgi Dimitrov (1933-1949). New Haven 2003.

Brasó, C., "Las Aduanas Marítimas de China y el comercio sino-español, 19001930". Revista de Historia Industrial. Economía y Empresa 26 (2017), pp. 109-143.

Brown, K., "Lee Huan obituary. Former premier of Taiwan who played a key role in the 1970s". The Guardian, 12 de diciembre de 2010, https://www. theguardian.com/world/2010/dec/12/lee-huan-obituary (último acceso: 05/07/2020).

Central Intelligence Agency (CIA), Biographic Sketch Chiang Ching-Kuo (sic) (1 de enero de 1962), https://www.cia.gov/library/readingroom/document/0000608234 (último acceso: 15/07/2020).

Central Intelligence Agency (CIA), Current Intelligence Bulletin-1955/09/21 (21 de septiembre de 1955), p. 5, https://www.cia.gov/library/readingroom/document/03000955 (último acceso: 15/07/2020).

Central Intelligence Agency (CIA), Taiwan After Chiang Ching-Kuo: A Speculative Analysis (1 de septiembre de 1982), https://www.cia.gov/ library/readingroom/docs/CIA-RDP84S00554R000100140003-0.pdf (último acceso: 01/06/2020).

103. Wang，Z., “小蔣辭世30年過半民眾仍感念政績! 歷任總統蔣經國貢獻最大 30 años después de la muerte de Chiang más de la mitad de los encuestados todavía extraña sus logros. Chiang Ching-kuo hizo la mayor contribución a la Presidencia [traducción propia]". China Times, 12 de enero de 2018, https://www.chinatimes.com/newspapers/20180112000497260118?chdtv (último acceso: 29/10/2020). Dai, L., “美麗島民調 : 2018年6月國政民調 “Sondeo Meilidao: encuesta de política nacional de junio de 2018" [traducción propia]. Formosa. Meilidao Dianzibao, 29 de junio de 2018, http://www.my-formosa.com/DOC_135525.htm (último acceso: 29/10/2020). 
Chen, C., "La revolución fascista china: una alternativa de defensa del país antes del estallido de la Segunda Guerra Sino-Japonesa", Herrera-Feligreras, A., et alii, España y China 1937-2017. 80 aniversario del Internacionalismo Antifascista. Granada 2017, pp. 35-53.

Chen, C., Radicalización del nacionalismo chino moderno: orígenes y desarrollo del fascismo chino. El caso de las organizaciones fascistas del Guomintang: la Sociedad Lixingshe y el Movimiento de la Nueva Vida (1927-1937). Barcelona 2014.

Chen, H., “我國經濟自由化之探討 A Study on Economic Liberalization Policy in Taiwan". 經濟研究Economic Research 13 (2013), pp. 183-206.

Chen, M., “228被扭曲的歷史集體記憶 Incidente del 28 de Febrero: una memoria colectiva histórica distorsionada [Traducción propia]". Straits Review Monthly 172 (2005), https://www.haixia-info.com/articles/4234.html (último acceso: 20/07/2020).

Cheng, T., "Democratizing the Quasi-Leninist Regime in Taiwan". World Politics 41 (1989), pp. 471-499.

Chiang, C., "Chiang: Constitution To Determine His Successor: President Bars Military Rule, No Family Member To Be Elected". Free China Journal, 30 de diciembre de 1985 [A partir de 2009, esta publicación pasó a llamarse Taiwan Today], https://web.archive.org/web/20160808114036/http:// www.taiwantoday.tw/ct.asp?xltem=117721\&CtNode=103 (último acceso: 02/06/2020).

Chiu, T., 蔣經國人格特質與臺灣政治發展 (1972-1988) Chiang Ching-kuo's Personality Characteristics and Taiwan Political Development (1972-1988) [Tesis doctoral]. Taipei 2008.

Culp, R., "Rethinking Governmentality: Training, Cultivation, and Cultural Citizenship in Nationalist China". The Journal of Asian Studies 65 (2006), pp. 529-554.

Dai, L., “美麗島民調：2018年6月國政民調 “Sondeo Meilidao: encuesta de política nacional de junio de 2018" [traducción propia]. Formosa. Meilidao Dianzibao, 29 de junio de 2018, http://www.my-formosa.com/DOC_135525. htm (último acceso: 29/10/2020).

Del Río Morillas, M. A., "La conexión anticomunista sino-española: Chiang Kaishek y Franco (1953-1973)". Dictatorships \& Democracies. Jounal of History and Culture 5 (2017), pp. 249-281, https://doi.org/10.7238/dd.v0i5.3139

Dirlik, A., "The Ideological Foundations of the New Life Movement: A Study in Counterrevolution". The Journal of Asian Studies 34 (1975), pp. 945-980.

Fairbank, J. K., China, una nueva historia. Barcelona 1996.

Fell, D., Government and Politics in Taiwan. London 2012.

Fields, K. J., "KMT, Inc. Party Capitalism in A Developmental State". JPRI Working Paper 47, http://www.jpri.org/publications/workingpapers/wp47.html (último acceso: 12/07/2020). 
Garver, J., "Taiwan's Russian Option: Image and Reality". Asian Survey 18 (1978), pp. 751-766.

Golub, P., East Asia's Reemergence. Cambridge 2016.

Gray, K., "Taiwan and the geopolitics of late development". The Pacific Review 24 (2011), pp. 577-599.

Greitens, S. C., Dictators and Their Secret Police: Coercive Institutions and State Violence. Cambridge 2016.

Griffin, R., Modernismo y fascismo. La sensación de comienzo bajo Mussolini y Hitler. Madrid 2010.

Herrera-Feligreras, A., España y China (1973-2005). Del reconocimiento diplomático a la Alianza Estratégica. Barcelona 2015.

Herrera-Feligreras A. y Lu, Y., "Espacios punitivos, entre el olvido y la memoria. El caso del Penal de San Cristóbal y el Centro de Detención de Jing-Mei", Oliver, P. y Urda, J. C., La prisión y las instituciones punitivas en la investigación histórica. Cuenca 2014, pp. 885-902.

Hickey, D. V. H., Foreign Policy Making in Taiwan. From Principle to Pragmatism. London 2007.

Hobsbawm, E., Historia del siglo XX, 1914-1991. Barcelona 2003.

Hsiung, J., "Diplomacy against Adversity: Foreign Relations under Chiang Chingkuo". Asian Affairs: An American Review 27 (2000), pp. 111-123.

$\mathrm{Hu}, \mathrm{C} .$, "Taiwan's geopolitics and Chiang Ching-Kuo's decision to democratize Taiwan". Stanford Journal of East Asian Affairs 5 (2005), pp. 26-44.

Huang，C., 日據五十年統治對光復後台灣之影響 La influencia de los 50 años de la ocupación japonesa sobre Taiwán tras su retorno a China [traducción propia]. Taipei 2002.

Kapur, H., Taiwan in a Changing World: Search for Security. Bloomington 2004.

Kennedy, S., "AMBASSADOR THOMAS P. SHOESMITH". Studies and Training Foreign Affairs Oral History Project, 9 de diciembre de 1991, https:// www.adst.org/OH\%20TOCs/Shoesmith,\%20Thomas.toc.pdf (último acceso: 15/07/2020).

Kung, I.,「外來政權」與本土社會 El “régimen extranjero" y la sociedad civil taiwanesa [traducción propia]. Taipei 1998.

Lee, K., 蔣經國與後蔣時代的內閣政治䓯英 (1972 1993年) Elites políticas del gabinete en la era post Chiang Ching-kuo (1972-1993) [Traducción propia]. Taipei 2014.

Lee, S., 快讀台灣史 Breve historia de Taiwán [traducción propia]. Taipei 2002.

Leng, S., Chiang Ching-Kuo's Leadership in the Development of the Republic of China on Taiwan. Lanham 1993.

Li, L., Madame Chiang Kai-shek: China's Eternal First Lady. New York 2006. 
Lin, H., "Taiwan's Cold War in Southeast Asia | Wilson Center". CWIHP E-Dossier 70 (2016), https://www.wilsoncenter.org/publication/taiwans-cold-warsoutheast-asia (último acceso: 01/07/2020).

Liu, F., "Taiwan's Security Policy Since The Cold War Era: A Review Of External Military Assistance And The Development Of Indigenous Defence Industry". History Of Global Arms Transfer 9 (2020), pp. 3-25.

Lu, Y., Taiwán. Historia Política e Identidad. Barcelona 2010.

Manthorpe, J., Forbidden Nation: A History of Taiwan. New York 2005.

Marks, T. A., Counterrevolution in China: Wang Sheng and the Kuomintang. New York 2014.

Martínez-Robles, D., "Reescrituras de la historia: China en las estrategias discursivas de la historiografía occidental". Gerónimo de Uztariz 25 (2009), pp. 47-66.

Murai, Tomoko. "La formación de la identidad taiwanesa en el siglo XX". México y la Cuenca del Pacífico 2 (1999), pp. 24-27.

Pantsov, A., "From Students to Dissidents. The Chinese Trotskyists in Soviet Rusia", https://www.marxists.org/history/etol/writers/pantsov/1994/china/studiss3. html (último acceso: 15/07/2020).

Pantsov, A., The Bolsheviks and the Chinese Revolution 1919-1927. London 2013.

Peng, H., 台灣政治發展 Political development in Taiwan. Taipei 2003.

Radek, K., Las vías y las fuerzas motrices de la Revolución Rusa. Madrid 1976.

Ren, Y., “台灣地區基層民主選舉中的「黑金政治」Política de dinero negro en las elecciones democráticas a nivel local en Taiwán [traducción propia]". Twenty-First Century [Versión digital] 38 (2005), http://www.cuhk.edu.hk/ ics/21c/media/online/0411019.pdf (último acceso: 28/10/2020).

Rigger, S., From opposition to power. Taiwan's Democratic Progressive Party. London, 2001.

Rigger, S., Why Taiwan Matters: Small Island, Global Powerhouse. Lanham 2011.

Sang, X., "Power, Interests, and Internal Factors. A Neoclassical Realist Perspective on the Taiwan Issue". Ghent 2017, https://biblio.ugent.be/publication/8534625/ file/8534683.pdf (último acceso: 03/06/2020).

Share, M., "From Ideological Foe to Uncertain Friend: Soviet Relations with Taiwan, 1943-82". Cold War History 3 (2003), pp. 1-34.

Shih, H. y Chung, J., "Xi Jinping Speech: Xi's position differs from the KMT's: Ma Ying-jeou". Taipei Times, 4 de enero de 2019, http://www.taipeitimes.com/ News/taiwan/archives/2019/01/04/2003707321 (último acceso: 07/06/2020).

Shimoda, K., "Chiang Ching-Kuo's 'Desired' Return to China in 1937. Viewed from the Document of the USSR". Journal of the Graduate School of AsiaPacific Studies 31 (2016), pp. 19-38. 
Spence, J. D., En busca de la China Moderna. Barcelona 2011.

Standing Committee of the Fifth National People's Congress, Message to Compatriots in Taiwan, 1 de enero de 1979, http://www.china.org.cn/english/7943. htm (último acceso: 03/07/2020).

Taipei Times, "Ma praises Lee Huan for role in political reforms". Taipei Times, el 20 de diciembre de 2010, http://www.taipeitimes.com/News/taiwan/ archives/2010/12/20/2003491431 (último acceso: 12/07/2020).

Taylor, J., The Generalissimo's Son: Chiang Ching-Kuo and the Revolutions in China and Taiwan. Cambridge 2009.

Tien, H., The Great Transition. Political and Social Change in the Republic of China. Stanford 1989.

Tubilewicz, C., "Taiwan and the Soviet Union During the Cold War: Enemies or Ambiguous Friends?". Cold War History 5 (2005), pp. 75-86.

United Daily News, 15.X.1987, p. 1.

Wakeman F., "A Revisionist View of the Nanjing Decade: Confucian Fascism". The China Quarterly 150 (1997), pp. 395-432.

Wang, Z., “小蔣辭世30年過半民眾仍感念政績！歷任總統蔣經國貢獻最大 30 años después de la muerte de Chiang más de la mitad de los encuestados todavía extraña sus logros. Chiang Ching-kuo hizo la mayor contribución a la Presidencia [traducción propia]". China Times, 12 de enero de 2018, https:// www.chinatimes.com/newspapers/20180112000497-260118?chdtv (último acceso: 29/10/2020).

Wei, H., “蔣經國對毛人鳳的最後一擊 El último golpe de Chiang Ching-kuo a Mao Renfeng [Traducción propia]". NEWSSC.org (四川新新闻网), http:// www.chinanews.com/tw/lscq/news/2007/09-04/1018588.shtml (último acceso: 20/07/2020).

Winckler, E. A. y Greenhalgh, S. (eds.), 臺灣政治經濟學諸論辯析 Contending Approaches to the Political Economy of Taiwan. Taipei 1994.

Wu, P., "Forgotten first lady served as model traditional wife", Taiwan Times, 18 de mayo de 2003. http://www.taipeitimes.com/News/taiwan/ archives/2003/05/18/0000211042 (último acceso: 29/10/2020).

Zhai, X. y Xiao, R., "Shifting political calculation: the secret Taiwan-Soviet talks, 1963-1971". Cold War History 15 (2015), pp. 533-556. 